\title{
Roles of Metal-Free Materials in Photoelectrodes for Water Splitting
}

Xiaochun Li, Jiawen Wang, Yuanxing Fang, * Hong Zhang, Xianzhi Fu and Xinchen Wang*

State Key Laboratory of Photocatalysis on Energy and Environment, College of Chemistry, Fuzhou University, Fuzhou 350116, P. R. China.

E-mail: yxfang@fzu.edu.cn; xcwang@fzu.edu.cn. Website: http://wanglab.fzu.edu.cn.

KEYWORDS: Metal-free Materials, Photoelectrochemical Water splitting, Polymeric Carbon Nitride, Covalent Organic Framework, Boron Nitride. 


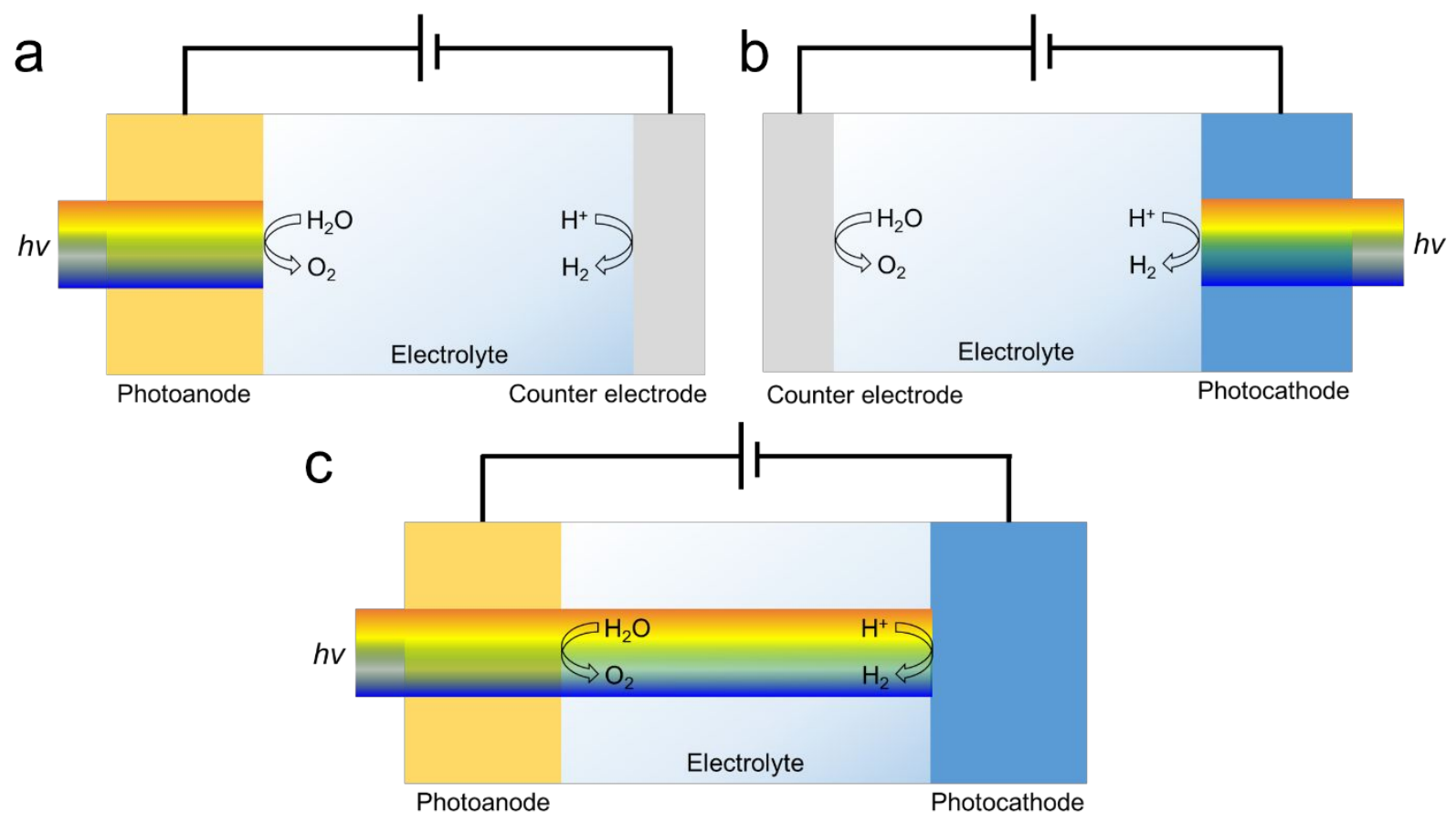

Figure S1. Illustrations of PEC systems. (a) Single photoanode system. (b) Single photocathode system. (c) Double-photoelectrode system.

\section{Discussion of Figure S1.}

Single photoelectrode system only consists of one electrode that can be active under light illumination, either photoanode (Figure S1a) or photocathode (Figure S1b) and the counter electrode at other side is generally based on electrocatalysis. Normally, n-type semiconductors were applied for photoanodes due to an upward band bending when immersed in an electrolyte solution, and p-type semiconductors were applied for photocathodes owing to the downward band bending in an electrolyte solution. When a single photoanode system is used, photoexcitation electrons and holes are formed on the surface of photoanode under light illumination. Holes at surface of photoanode drive water oxidation reaction, and meanwhile, photoexcitation electrons migrate to the cathode for water reduction reaction. Double- 
photoelectrode system is normally bias-free, and both photoelectrodes would be excited under light illumination (Figure S1c). Therefore, photoexcitation charges are formed on the surface of anode and cathode for water oxidation reaction and water reduction reaction, respectively.

In the photoanode system, the band edges of a photocatalyst are preferable to meet the potentials of redox reactions, and therefore it is possible to work without voltage bias. Voltage bias could be further applied to add thermodynamic energy. water oxidation reaction at photoanode can be either oxygen evolution reaction or hydrogen peroxide evolution reaction through 4-electron (Eq. S1) or 2-electron (Eq. S2) pathways, respectively, and thus the critical potentials for valence bands are $1.23 \mathrm{~V}$ or $1.77 \mathrm{~V}$ versus reversible hydrogen electrode ( $v s$. RHE). Meanwhile, the transferred electrons drive electrocatalytic hydrogen evolution reaction at cathode (Eq. S3), and thus the critical potential of conduction band (CB) is $0 \mathrm{~V}$ vs. reversible hydrogen electrode. ${ }^{1}$

$$
\begin{gathered}
\mathrm{H}_{2} \mathrm{O} \rightarrow \mathrm{O}_{2}+4 \mathrm{H}^{+}+4 \mathrm{e}^{-}, \mathrm{E}^{\circ}=+1.23 \mathrm{~V} \text { vs. RHE } \\
2 \mathrm{H}_{2} \mathrm{O} \rightarrow \mathrm{H}_{2} \mathrm{O}_{2}+2 \mathrm{H}^{+}+2 \mathrm{e}^{-}, \mathrm{E}^{\circ}=+1.77 \text { V vs. RHE } \\
2 \mathrm{H}^{+}+2 \mathrm{e}^{-} \rightarrow \mathrm{H}_{2}, \mathrm{E}^{\circ}=0 \text { V vs. RHE }
\end{gathered}
$$

With respect to the single photoelectrode system, the requirements of photocatalyst for double-photoelectrode system are sophisticated (Figure S1c). In term of band edges, the VB of photoanode and $\mathrm{CB}$ of photocathode must meet the potentials for oxidation and reduction reactions, respectively. In addition, the bandgaps of photocatalysts at photoanode and photocathode should be coordinated to each other, since the incident light is generally absorbed 
by one photoelectrode, and the other one absorbs the penetrated light. The difference of the photocatalyst bandgap is preferable to be large, and thus to maximize the visible light absorption. 


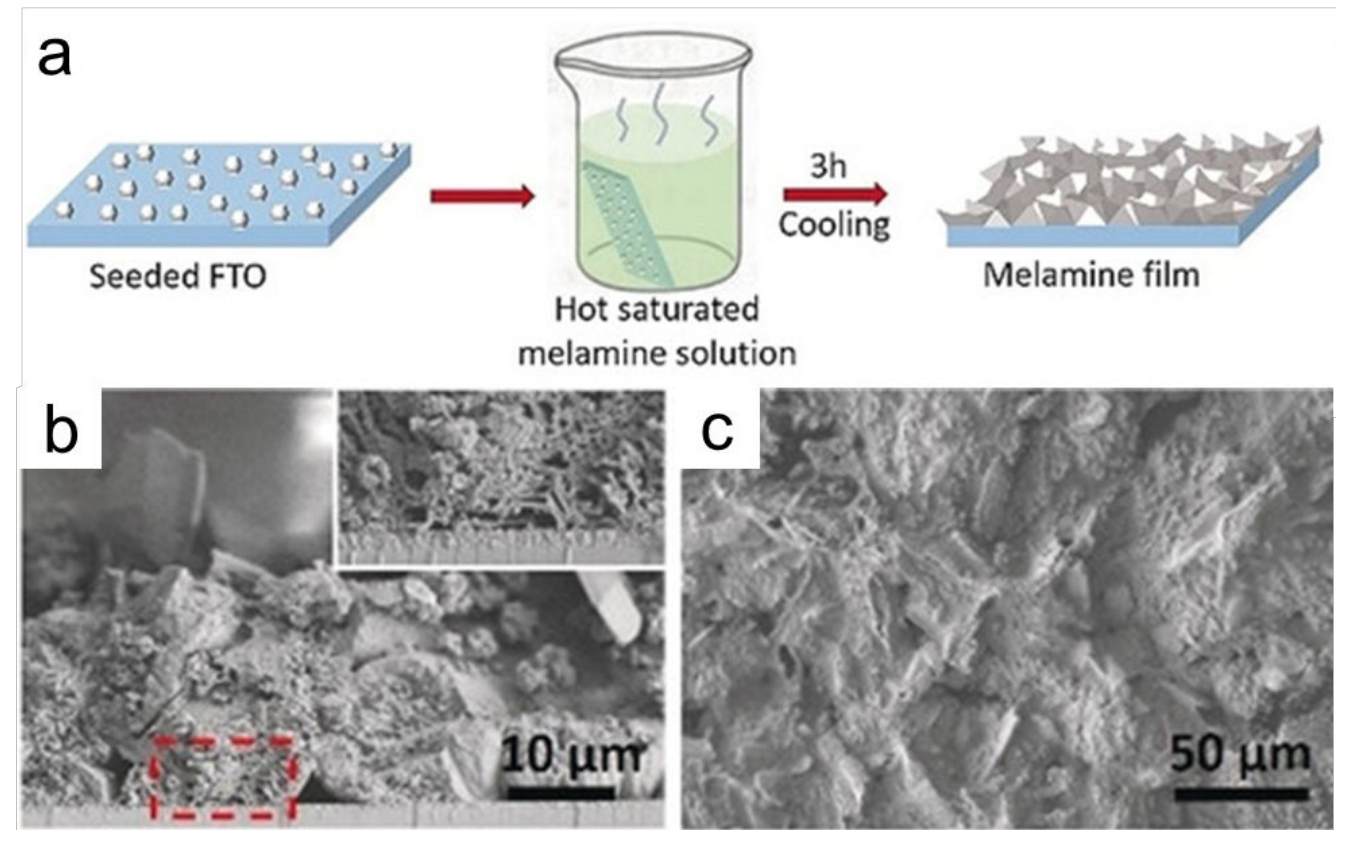

Figure S2. (a) Synthesis of the melamine films. (b) Cross-section and (c) top-view SEM image of the PCN film by annealing the melamine films, and area in red square is magnified as shown in the inset. Reproduced with permission from Ref. ${ }^{2}$, Copyright 2018, Wiley-VCH GmbH, Weinheim.

\section{Discussion of Figure S2.}

Solution-based synthesis was also developed to synthesize polymeric carbon nitride (PCN) films on fluorine doped tin oxide glass (Figure S2a). ${ }^{2}$ Generally, a substrate was seeded by PCN monomers, and the as-prepared substrate was immersed into a hot solution containing saturated melamine. The solution cooled down spontaneously to obtain melamine films, and PCN films can be achieved by thermal polymerization. The homogeneous seeding of PCN monomer would lead the growth of PCN films in uniform morphology as shown in Figure $\mathbf{S 2 b}$ and $\mathbf{S 2 c}$. As a result, the optimal PCN photoanode has shown a photocurrent density of ca. $116 \mu \mathrm{A} \mathrm{cm}^{-2}$ at 1.23 $\mathrm{V}$ vs. RHE under AM 1.5G illumination, and the stability can be preserved for 1 hour. This synthesis was expanded by using other precursors, including thiourea, urea, dicyandiamide, ammonium thiocyanate and guanidine carbonate. ${ }^{3}$ 


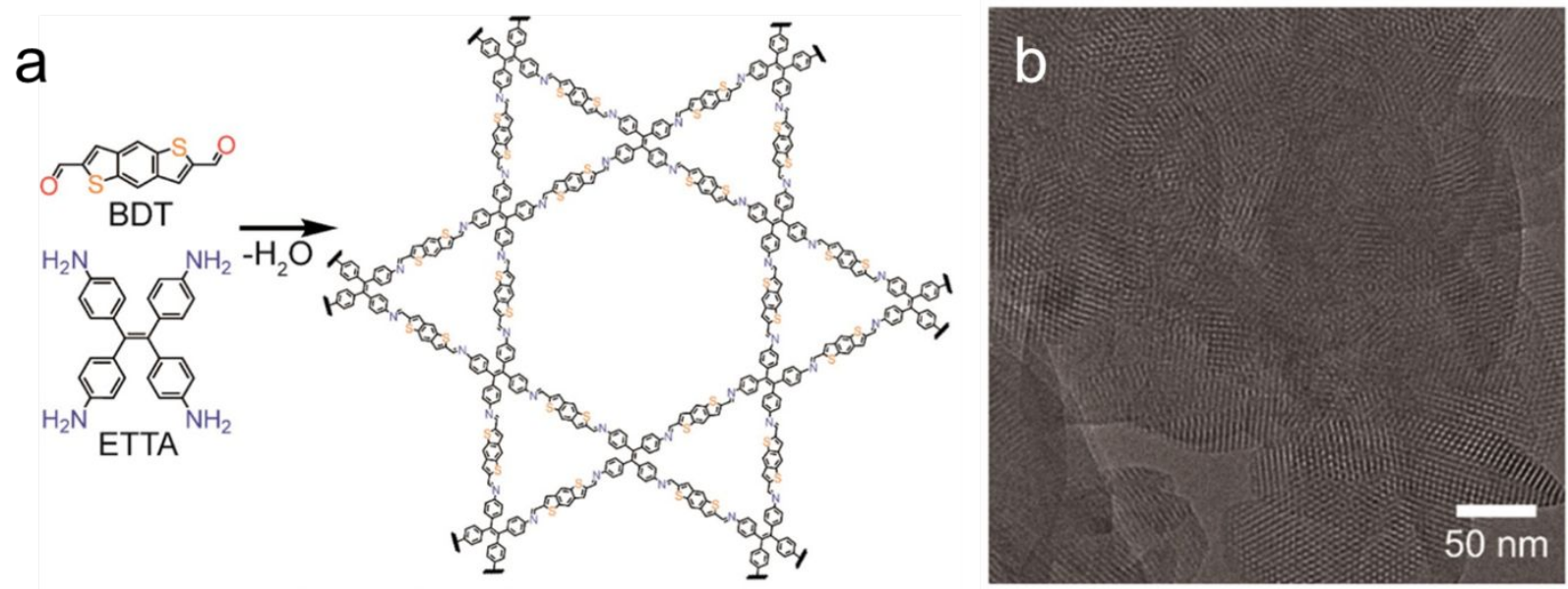

Figure S3. (a) Synthesis of a BDT-ETTA COF. (b) TEM image of the high crystalline BDT-ETTA COF. Reproduced with permission from Ref. ${ }^{4}$, Copyright 2018, American Chemical Society.
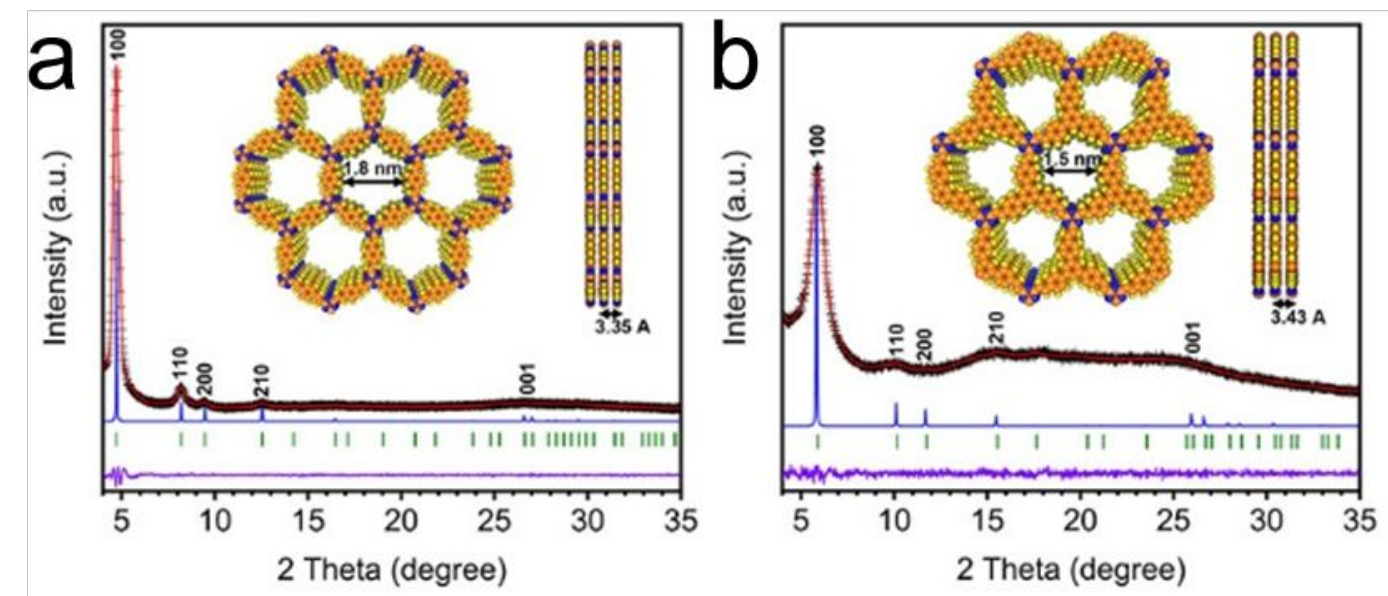

Figure S4. Powder X-ray diffraction patterns of (a) $g-\mathrm{C}_{18} \mathrm{~N}_{3}-\mathrm{COF}$ and (b) $g-\mathrm{C}_{33} \mathrm{~N}_{3}-\mathrm{COF}$. Insets are structural models of $\mathrm{g}-\mathrm{C}_{18} \mathrm{~N}_{3}-\mathrm{COF}$ and $\mathrm{g}-\mathrm{C}_{33} \mathrm{~N}_{3}-\mathrm{COF}$ in eclipsed (AA) stacking. Reproduced with permission from Ref. ${ }^{5}$, Copyright 2019, American Chemical Society. 


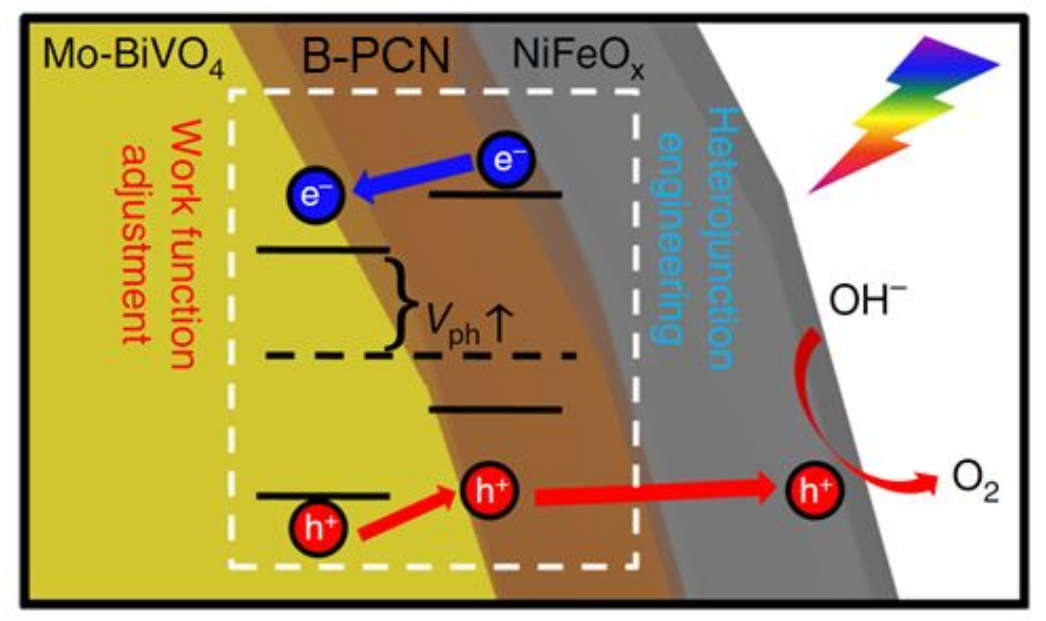

Figure S5. The dynamics of the photoexcitated charges on $\mathrm{NiFeO}_{\mathrm{x}} / \mathrm{B}-\mathrm{PCN} / \mathrm{Mo}-\mathrm{BiVO}_{4}$ photoanode. Reproduced with permission from Ref. ${ }^{6}$, Copyright 2019, Springer Nature Publishing AG.

\section{Discussion of Figure S5.}

PCN materials were demonstrated to coat on $\mathrm{BiVO}_{4}$ films as heterostructure photocatalytic layer, ${ }^{6}$ since the bandgap of $\mathrm{BiVO}_{4}$ and $\mathrm{PCN}$ are ca. 2.4 and $2.6 \mathrm{eV}$, respectively. When PCN films are coated on the $\mathrm{BiVO}_{4}$ material, the light region with wavelength smaller than $480 \mathrm{~nm}$ can excite PCN layer, and those with the wavelength larger than $480 \mathrm{~nm}$ would penetrate over the PCN to excite $\mathrm{BiVO}_{4}$. As a noble example, the structure of photoanode is shown in Figure S5. Specifically, Mo-doped $\mathrm{BiVO}_{4}$ was used as the bottom layer, and it was coated by Boron doped PCN (B-PCN) as photocatalytic layer. $\mathrm{NiFeO}_{\mathrm{x}}$ was loaded on $\mathrm{B}-\mathrm{PCN}$ as co-catalyst to prepare a photoanode. B-PCN among the photocatalytic layer not only improves the light absorption, but also forms type II heterostructure with $\mathrm{BiVO}_{4}$ to improve charge separation and transfer. This photoanode has achieved a PD of $5.93 \mathrm{~mA} \mathrm{~cm}^{-2}$ at $1.23 \mathrm{~V}$ vs. RHE under AM 1.5G illumination. The production rates of $\mathrm{H}_{2}$ and $\mathrm{O}_{2}$ gases at $0.54 \mathrm{~V} v s$. RHE were ca. $77.5 \mu \mathrm{mol} \mathrm{cm}{ }^{-2}$ $\mathrm{h}^{-1}$ and $33.6 \mu \mathrm{mol} \mathrm{cm} \mathrm{cm}^{-2} \mathrm{~h}^{-1}$, respectively. 


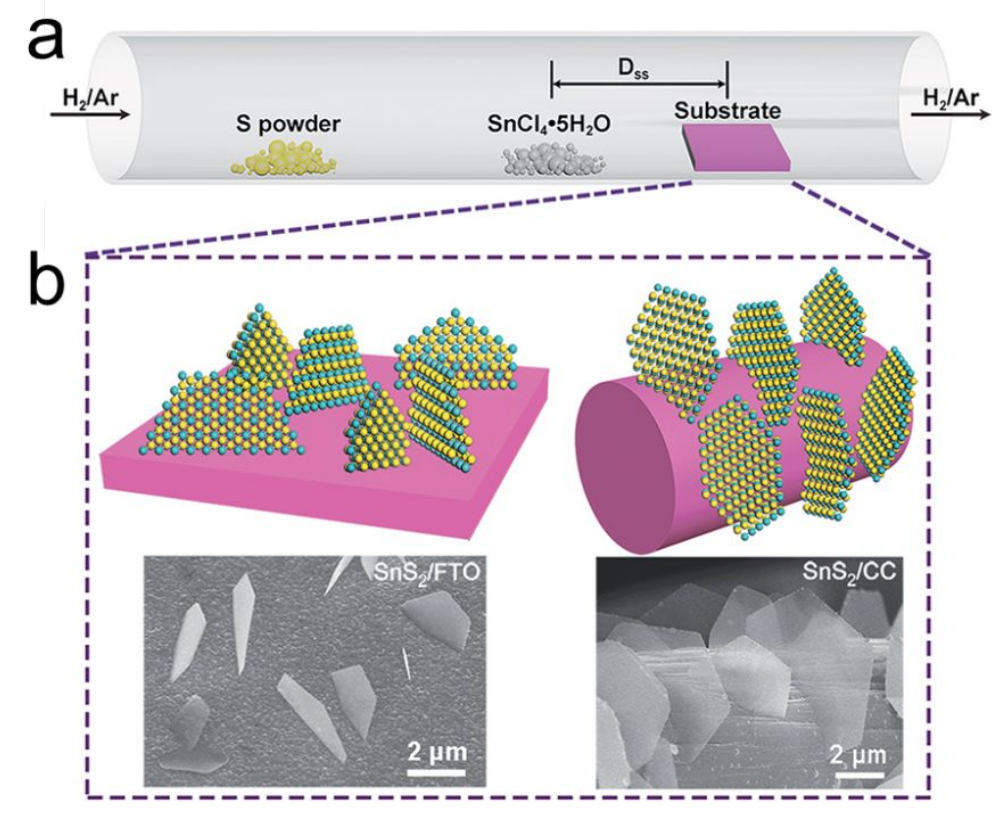

Figure S6. Schematic illustration for the design and synthesis of vertically aligned $2 \mathrm{D} \mathrm{SnS}_{2}$ nanosheets. (a) Schematic for CVD growth. (b) Morphological models and SEM images of vertically aligned 2D $\mathrm{SnS}_{2}$ nanosheets grown on planar/curved substrates. Reproduced with permission from Ref.', Copyright 2017, The Royal Society of Chemistry. 

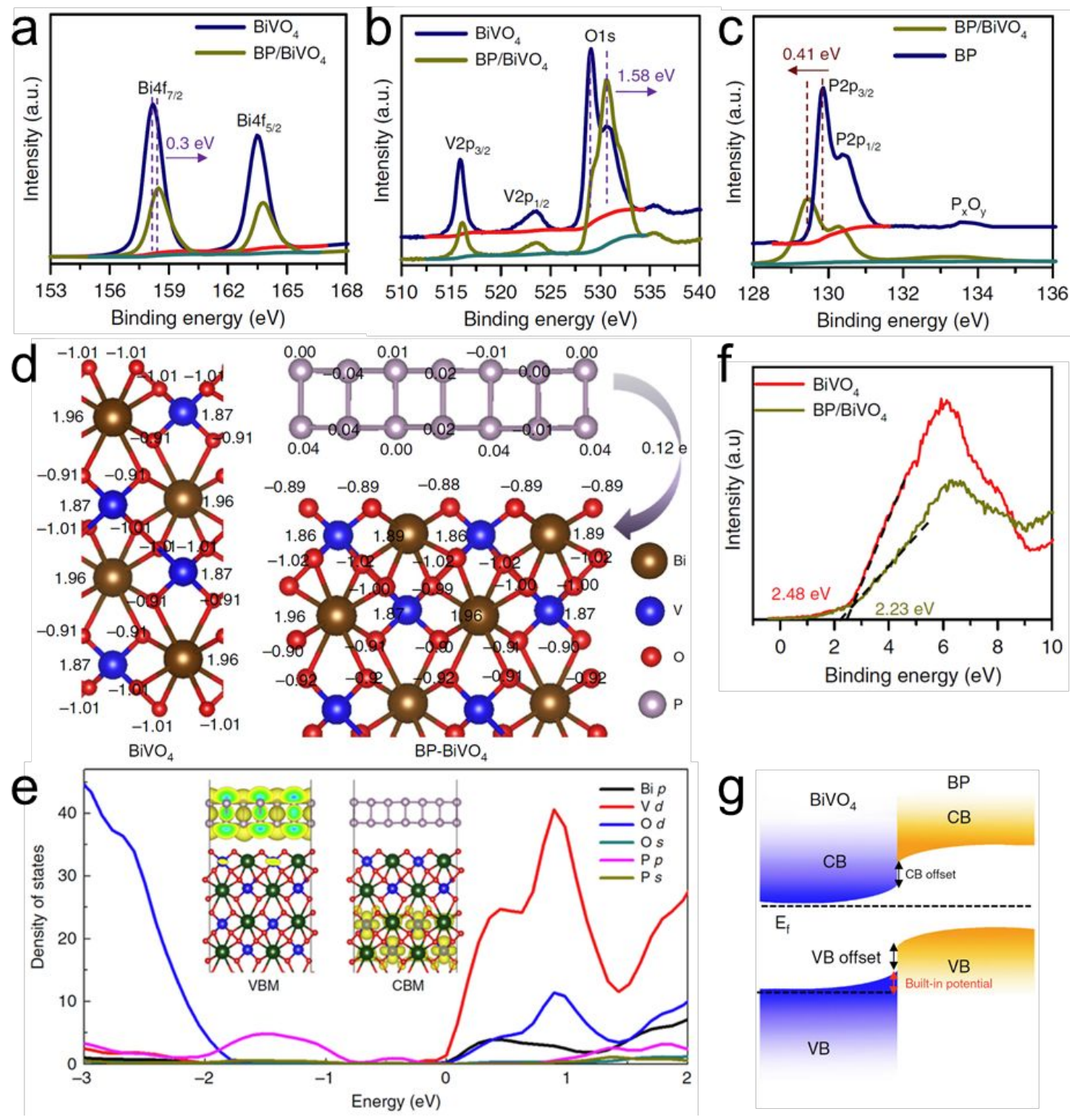

Figure S7. (a) Bi 4f, (b) V 2p and O 1s XPS of $\mathrm{BiVO}_{4}$ and $\mathrm{BP} / \mathrm{BiVO}_{4}$ photoanodes. (c) P 2p XPS of BP and $\mathrm{BP} / \mathrm{BiVO}_{4}$ photoanode. (d) Theoretical calculation of the charge density difference between $\mathrm{BiVO}_{4}$ and $\mathrm{BP} / \mathrm{BiVO}_{4}$. (e) Density of states diagram for $\mathrm{BP} / \mathrm{BiVO}_{4}$ heterojunction. (f) $\mathrm{VB}$ of $\mathrm{BiVO}_{4}$ and $\mathrm{BP} / \mathrm{BiVO}_{4}$ heterojunction by XPS. (g) Band structure of $\mathrm{BP} / \mathrm{BiVO}_{4}$ photoanode. Reproduced with permission from Ref. ${ }^{8}$ Copyright 2019, Springer Nature Publishing AG.

\section{Discussion of Figure S7.}


2D black phosphorene (BP) can be applied as co-catalyst for photoanodic OER. Generally, BP is p-type semiconductors with high hole mobility $\left(1000 \mathrm{~cm}^{2} \mathrm{~V}^{-3} \mathrm{~s}\right)$, and thus potential to act as charge acceptor in a photoanode. ${ }^{9}$ As a unique example, 4 layered BP was loaded on $\mathrm{BiVO}_{4}$ films, and co-catalyst of $\mathrm{NiOOH} / \mathrm{FeOOH}$ was coated on the surface of BP. In this work, the ultrathin BP was prepared by ultrasonic exfoliation of bulky BP, which was suspended in isopropanol as ink. ${ }^{10}$ Subsequently, $\mathrm{BiVO}_{4}$ photoanode was immersed into the BP ink, and BP would be absorbed on the $\mathrm{BiVO}_{4}$ spontaneously. Photoelectron-deposition was applied to load $\mathrm{NiOOH}$ on BP-coated $\mathrm{BiVO}_{4}{ }^{11}$ XPS spectra of Bi 4f, O 1s and P 2p are shown in Figure S7a to S7c, respectively. It is obvious when $\mathrm{BP}$ was loaded on $\mathrm{BiVO}_{4}$, and the binding energies were shifted, revealing the chemical integration between $\mathrm{BP}$ and $\mathrm{BiVO}_{4}$. This integration is also stimulated by theoretical calculation as shown in Figure S7d and S7e, and it makes agreement with the XPS results. The VB of the BP and hybrid were studied by XPS as shown in Figure S7f, and correspondingly, the band structures are shown in Figure S7g. As a result, the tri-layered photoanode has achieved a PD of ca. $4.48 \mathrm{~mA} \mathrm{~cm}^{-2}$ at $1.23 \mathrm{~V}$ vs. RHE under AM 1.5G illumination, which is ca. 1.5 times to the $\mathrm{NiOOH} / \mathrm{BiVO}_{4}$ photoanode. 
Table S1. Photoelectrochemical performances of single-layered polymeric carbon nitride photoanodes.

\begin{tabular}{|c|c|c|c|c|c|c|}
\hline Catalyst & Preparation method & $\begin{array}{c}\text { Photocurrent } \\
\text { density }(\mu \mathrm{A} \\
\left.\mathrm{cm}^{-2}\right)\end{array}$ & $\begin{array}{l}\text { Potential } \\
\text { vs RHE (V) }\end{array}$ & Electrolyte & Light source & $\begin{array}{c}\text { Corresponding } \\
\text { Author (Ref.) }\end{array}$ \\
\hline $\begin{array}{c}\mathrm{CN}_{\mathrm{T}} \\
\text { photoanode }\end{array}$ & $\begin{array}{l}\text { Seeded crystallization } \\
\text { of CN monomers }\end{array}$ & 266 & 1.23 & $0.1 \mathrm{M} \mathrm{KOH}$ & $\begin{array}{c}100 \mathrm{~mW} \mathrm{~cm}{ }^{-2}, \mathrm{AM} \\
1.5 \mathrm{G} \text { (Newport) }\end{array}$ & 3 \\
\hline CNP films & $\begin{array}{l}\text { Evaporation } \\
\text { polymerization }\end{array}$ & 230 & 1.23 & $\begin{array}{c}0.5 \mathrm{M} \\
\mathrm{Na}_{2} \mathrm{SO}_{4}\end{array}$ & Xe lamp, AM 1.5G & 12 \\
\hline $\mathrm{CN}$ films & $\begin{array}{l}\text { Thermal vapor } \\
\text { condensation }\end{array}$ & 228.2 & 1.23 & $\begin{array}{c}0.2 \mathrm{M} \\
\mathrm{Na}_{2} \mathrm{SO}_{4}\end{array}$ & $\begin{array}{c}150 \mathrm{~W} \text { Xe lamp, } \\
\text { AM 1.5G (Newport) }\end{array}$ & 13 \\
\hline $\begin{array}{l}\text { g-CN PNR array } \\
\text { photoanode }\end{array}$ & $\begin{array}{c}\text { Thermal } \\
\text { polycondensation } \\
\text { using AAO template }\end{array}$ & 120.5 & 1.23 & $\begin{array}{c}0.1 \mathrm{M} \\
\mathrm{Na}_{2} \mathrm{SO}_{4}\end{array}$ & $\begin{array}{c}500 \mathrm{~W} \text { Xe lamp, } \\
\text { AM } 1.5 \mathrm{G}\end{array}$ & 14 \\
\hline $5 \mathrm{p}-\mathrm{PCN}$ films & $\begin{array}{l}\text { Molten mediate } \\
\text { polymerization }\end{array}$ & ca. 120 & 1.23 & $\begin{array}{l}1.0 \mathrm{M} \\
\mathrm{NaOH}\end{array}$ & $\begin{array}{c}100 \mathrm{~mW} \mathrm{~cm}{ }^{-2}, \mathrm{AM} \\
1.5 \mathrm{G} \text { (Newport) }\end{array}$ & 15 \\
\hline $\begin{array}{l}\text { phosphorylated } \\
\text { PCN films }\end{array}$ & $\begin{array}{l}\text { Molten mediate } \\
\text { polymerization, } \\
\text { immersion method }\end{array}$ & ca. 120 & 1.23 & $\begin{array}{l}1.0 \mathrm{M} \\
\mathrm{NaOH}\end{array}$ & $\begin{array}{c}100 \mathrm{~mW} \mathrm{~cm}-2, \mathrm{AM} \\
1.5 \mathrm{G} \text { (Newport) }\end{array}$ & 16 \\
\hline SOCN-75 films & $\begin{array}{l}\text { Thermal vapor } \\
\text { condensation }\end{array}$ & 119.2 & 1.23 & $\begin{array}{c}0.1 \mathrm{M} \\
\mathrm{Na}_{2} \mathrm{SO}_{4}\end{array}$ & $\begin{array}{c}150 \mathrm{~W} \text { Xe lamp, } \\
\text { AM 1.5G (Newport) }\end{array}$ & 17 \\
\hline CN films & $\begin{array}{l}\text { Seeded crystallization } \\
\text { of CN monomers }\end{array}$ & 116 & 1.23 & $0.1 \mathrm{M} \mathrm{KOH}$ & $\begin{array}{c}100 \mathrm{~mW} \mathrm{~cm}{ }^{-2}, \mathrm{AM} \\
1.5 \mathrm{G} \text { (Newport) }\end{array}$ & 2 \\
\hline PCN films & $\begin{array}{l}\text { Molten mediate } \\
\text { polymerization }\end{array}$ & 100 & 1.23 & $\begin{array}{l}1.0 \mathrm{M} \\
\mathrm{NaOH}\end{array}$ & $\begin{array}{c}100 \mathrm{~mW} \mathrm{~cm}{ }^{-2}, \mathrm{AM} \\
1.5 \mathrm{G} \text { (Newport) }\end{array}$ & 18 \\
\hline Thio-CA films & $\begin{array}{l}\text { Thermal vapor } \\
\text { condensation }\end{array}$ & 96.2 & 1.23 & $\begin{array}{c}0.2 \mathrm{M} \\
\mathrm{Na}_{2} \mathrm{SO}_{4}\end{array}$ & $\begin{array}{c}150 \mathrm{~W} \text { Xe lamp, } \\
\text { AM } 1.5 \mathrm{G} \text { (Newport) }\end{array}$ & 19 \\
\hline
\end{tabular}


Table S1. (continued) Photoelectrochemical performances of single-layered polymeric carbon nitride photoanodes.

\begin{tabular}{|c|c|c|c|c|c|c|}
\hline Catalyst & Preparation method & $\begin{array}{c}\text { Photocurrent } \\
\text { density }(\mu \mathrm{A} \\
\left.\mathrm{cm}^{-2}\right)\end{array}$ & $\begin{array}{c}\text { Potential } \\
\text { vs RHE (V) }\end{array}$ & Electrolyte & Light source & $\begin{array}{c}\text { Corresponding } \\
\text { Author (Ref.) }\end{array}$ \\
\hline $\begin{array}{l}\text { framework } g-\mathrm{C}_{3} \mathrm{~N}_{4} \\
\text { films }\end{array}$ & $\begin{array}{c}\text { Thermal vapor } \\
\text { liquid- } \\
\text { polymerization }\end{array}$ & 89 & 1.1 & $\begin{array}{c}0.1 \mathrm{M} \\
\mathrm{Na}_{2} \mathrm{SO}_{4}\end{array}$ & $\begin{array}{c}100 \mathrm{~mW} \mathrm{~cm}{ }^{-2}, \mathrm{AM} \\
1.5 \mathrm{G}\end{array}$ & 20 \\
\hline $\begin{array}{l}\text { DPCN/NRGO/NiFe- } \\
\text { LDH photoanode }\end{array}$ & $\begin{array}{l}\text { Electrophoretic } \\
\text { deposition }\end{array}$ & 72.9 & 1.22 & $\begin{array}{r}0.01 \mathrm{M} \\
\mathrm{Na}_{2} \mathrm{SO}_{4}\end{array}$ & $\begin{array}{c}150 \text { W Xe lamp, } \\
\text { AM 1.5G }\end{array}$ & 21 \\
\hline $\mathrm{CN}-\mathrm{rGO}_{0.5}$ films & $\begin{array}{l}\text { Doctor-blade } \\
\text { technique }\end{array}$ & 72 & 1.23 & $\begin{array}{l}0.1 \mathrm{M} \\
\mathrm{KOH}\end{array}$ & $\begin{array}{c}100 \mathrm{~mW} \mathrm{~cm}{ }^{-2}, \mathrm{AM} \\
1.5 \mathrm{G} \text { (Newport) }\end{array}$ & 22 \\
\hline $3 \% \mathrm{Ni}-\mathrm{CN}$ & $\begin{array}{l}\text { liquid mediated } \\
\text { pathway }\end{array}$ & 69.8 & 1.23 & $\begin{array}{l}0.1 \mathrm{M} \\
\mathrm{KOH}\end{array}$ & $\begin{array}{c}100 \mathrm{~mW} \mathrm{~cm}^{-2}, \mathrm{AM} \\
1.5 \mathrm{G} \text { (Newport) }\end{array}$ & 23 \\
\hline $\begin{array}{l}\text { CN-dicyanamide } \\
\text { electrode }\end{array}$ & $\begin{array}{l}\text { two-step vapor } \\
\text { deposition }\end{array}$ & 63 & 1.23 & $\begin{array}{c}0.1 \mathrm{M} \\
\mathrm{Na}_{2} \mathrm{SO}_{4}\end{array}$ & $\begin{array}{l}300 \text { W Xe lamp, } \\
\text { AM 1.5G }\end{array}$ & 24 \\
\hline $\begin{array}{c}\mathrm{CN}_{\mathrm{MW}} \\
\text { photoelectrode }\end{array}$ & $\begin{array}{l}\text { Microwave-assisted } \\
\text { condensation }\end{array}$ & 38 & 1.23 & $0.1 \mathrm{M} \mathrm{KCl}$ & $\begin{array}{c}150 \text { W Xe lamp, } \\
\text { AM } 1.5 \mathrm{G}\end{array}$ & 25 \\
\hline CN@FTO-10\% & $\begin{array}{l}\text { Microcontact- } \\
\text { printing-assisted } \\
\text { access }\end{array}$ & 30.2 & 1.23 & $\begin{array}{c}0.2 \mathrm{M} \\
\mathrm{Na}_{2} \mathrm{SO}_{4}\end{array}$ & $\begin{array}{c}500 \mathrm{~W} \text { Xe lamp, } \\
\text { simulated solar } \\
\text { light source (AM } \\
1.5 \mathrm{G})\end{array}$ & 26 \\
\hline porous $\mathrm{CN}$ films & $\begin{array}{l}\text { Doctor-blade } \\
\text { technique }\end{array}$ & 12 & 1.23 & $\begin{array}{l}0.1 \mathrm{M} \\
\mathrm{KOH}\end{array}$ & $\begin{array}{c}100 \mathrm{~mW} \mathrm{~cm}^{-2}, \mathrm{AM} \\
1.5 \mathrm{G} \text { (Newport) }\end{array}$ & 27 \\
\hline g-CN-50 nanorods & VMFAIP system & 10.8 & 1.23 & $\begin{array}{c}0.5 \mathrm{M} \\
\mathrm{Na}_{2} \mathrm{SO}_{4}\end{array}$ & $\begin{array}{c}100 \mathrm{~mW} \mathrm{~cm}^{-2}, \mathrm{AM} \\
1.5 \mathrm{G}\end{array}$ & 28 \\
\hline $\mathrm{CNBC} / \mathrm{FTO}$ & $\begin{array}{l}\text { Solvothermal } \\
\text { method }\end{array}$ & 2 & 1.23 & $\begin{array}{c}0.5 \mathrm{M} \\
\mathrm{Na}_{2} \mathrm{SO}_{4}\end{array}$ & $\begin{array}{c}\text { Simulated solar } \\
\text { light source (AM } \\
1.5 \mathrm{G})\end{array}$ & 29 \\
\hline
\end{tabular}


Table S2. Photoelectrochemical performances of boron nitride and covalent organic frameworks based photoelectrodes.

\begin{tabular}{|c|c|c|c|c|c|c|}
\hline Catalyst & Preparation method & $\begin{array}{l}\text { Photocurrent } \\
\text { density }(\mu \mathrm{A} \\
\left.\mathrm{cm}^{-2}\right)\end{array}$ & $\begin{array}{c}\text { Potential } \\
\text { vs RHE (V) }\end{array}$ & Electrolyte & Light source & $\begin{array}{c}\text { Corresponding } \\
\text { Author (Ref.) }\end{array}$ \\
\hline BCN-700 & $\begin{array}{l}\text { Dip-coating } \\
\text { technique }\end{array}$ & ca. 0.9 & 0 & $\begin{array}{c}0.1 \mathrm{M} \\
\mathrm{Na}_{2} \mathrm{SO}_{4}\end{array}$ & 300 W Xe lamp & 30 \\
\hline $\begin{array}{l}\text { Powder-based C- } \\
\text { BN photoanode }\end{array}$ & - & ca. 2 & 1.60 & $\begin{array}{l}1.0 \mathrm{M} \\
\mathrm{NaOH}\end{array}$ & $\begin{array}{c}100 \mathrm{~mW} \mathrm{~cm}{ }^{-2}, \mathrm{AM} \\
1.5 \mathrm{G} \text { (Newport) }\end{array}$ & 31 \\
\hline C-BN1 & $\begin{array}{l}\text { Plasma enhanced } \\
\text { chemical vapor } \\
\text { deposition }\end{array}$ & ca. 120 & 1.60 & $\begin{array}{l}1.0 \mathrm{M} \\
\mathrm{NaOH}\end{array}$ & $\begin{array}{c}100 \mathrm{~mW} \mathrm{~cm}^{-2}, \mathrm{AM} \\
1.5 \mathrm{G} \text { (Newport) }\end{array}$ & 31 \\
\hline BCN thin films & $\begin{array}{l}\text { Vapor deposition } \\
\text { polymerization }\end{array}$ & - & - & - & - & 32 \\
\hline BBL photoanode & $\begin{array}{c}\text { Spray-coating } \\
\text { deposition method }\end{array}$ & $\begin{array}{c}0.23 \pm 0.02 \\
\mathrm{~mA} \mathrm{~cm}\end{array}$ & 1.23 & $\begin{array}{c}0.5 \mathrm{M} \\
\mathrm{Na}_{2} \mathrm{SO}_{3}(\mathrm{pH} \\
7)\end{array}$ & $\begin{array}{l}\text { Simulated solar light } \\
\text { source }\left(100 \mathrm{~mW} \mathrm{~cm}^{-}\right. \\
\left.{ }^{2}\right)\end{array}$ & 33 \\
\hline $\begin{array}{l}\text { BDT-ETTA } \\
\text { films }\end{array}$ & Solvothermal method & 0.9 & 0.4 & $\begin{array}{c}0.1 \mathrm{M} \\
\mathrm{Na}_{2} \mathrm{SO}_{4}\end{array}$ & $\begin{array}{l}\text { AM 1.5G solar } \\
\text { simulator }\end{array}$ & 4 \\
\hline $\begin{array}{l}\mathrm{g}-\mathrm{C}_{18} \mathrm{~N}_{3}-\mathrm{COF} \\
\text { films }\end{array}$ & $\begin{array}{l}\text { Drop-casting } \\
\text { technique }\end{array}$ & ca. 45 & 0.2 & $\begin{array}{c}0.2 \mathrm{M} \\
\mathrm{Na}_{2} \mathrm{SO}_{4}\end{array}$ & $\begin{array}{c}300 \mathrm{~W} \text { Xe lamp with } \\
\text { a } 420 \text { nm cut-off } \\
\text { filter }\end{array}$ & 5 \\
\hline $\begin{array}{l}\text { TAPB-TTB } \\
\text { films } \\
\text { TAPB-TTB } \\
\text { films }\end{array}$ & $\begin{array}{l}\text { Slurry-coated } \\
\text { technique }\end{array}$ & $\begin{array}{c}110 \\
35\end{array}$ & 0 & $\begin{array}{c}0.5 \mathrm{M} \\
\mathrm{Na}_{2} \mathrm{SO}_{4}\end{array}$ & $\begin{array}{l}300 \mathrm{~W} \text { Xe lamp with } \\
\text { a } 420 \mathrm{~nm} \text { cut-off } \\
\text { filter }\left(200 \mathrm{~mW} \mathrm{~cm}^{-2}\right)\end{array}$ & 34 \\
\hline $\begin{array}{l}\text { Tz-Th@Cu } \\
\text { BTT@Cu }\end{array}$ & $\begin{array}{l}\text { spray-coating } \\
\text { technique }\end{array}$ & $\begin{array}{l}6.8 \\
2.3\end{array}$ & $\begin{array}{l}0.6 \mathrm{~V} \text { vs. } \\
\mathrm{Ag} / \mathrm{AgCl}\end{array}$ & $\begin{array}{l}0.01 \mathrm{M} \\
\mathrm{Na}_{2} \mathrm{SO}_{4}\end{array}$ & $\begin{array}{l}300 \mathrm{~W} \text { Xe lamp } \\
\left(100 \mathrm{~mW} \mathrm{~cm} \mathrm{~cm}^{-2}\right)\end{array}$ & 35 \\
\hline $\begin{array}{l}\text { PTEB nanofiber } \\
\text { films }\end{array}$ & $\begin{array}{l}\text { Cu-surface-mediated } \\
\text { synthetic approach }\end{array}$ & ca. 10 & $0.3-0$ & $\begin{array}{l}0.01 \mathrm{M} \\
\mathrm{Na}_{2} \mathrm{SO}_{4}\end{array}$ & $\begin{array}{l}200 \text { W Xe lamp, } \\
\text { AM 1.5G (Newport) }\end{array}$ & 36 \\
\hline
\end{tabular}


Table S3. Photoelectrochemical performances of the heterostructure polymeric carbon nitride photoanodes.

\begin{tabular}{|c|c|c|c|c|c|c|c|}
\hline Catalyst & Preparation method & $\begin{array}{l}\text { Photocurrent } \\
\text { density }(\mu \mathrm{A} \\
\left.\mathrm{cm}^{-2}\right)\end{array}$ & $\begin{array}{l}\text { Gas } \\
\text { production } \\
\text { rate }\end{array}$ & $\begin{array}{c}\text { Potential } \\
\text { vs RHE (V) }\end{array}$ & Electrolyte & Light source & $\begin{array}{c}\text { Corresponding } \\
\text { Author (Ref.) }\end{array}$ \\
\hline $\begin{array}{c}\mathrm{CN}- \\
\mathrm{MR} / \mathrm{NiFeO}_{\mathrm{x}} \mathrm{H}_{\mathrm{y}} \\
\text { electrode }\end{array}$ & $\begin{array}{c}\text { Doctor-blade } \\
\text { technique, CVD-like } \\
\text { and solvothermal } \\
\text { process }\end{array}$ & $472 \pm 10$ & $\begin{array}{c}\mathrm{H}_{2}: \text { ca. } 9.0 \\
\mu \mathrm{mol} \mathrm{cm} \mathrm{cm}^{-2} \mathrm{~h}^{-1} \\
\mathrm{O}_{2}: \text { ca. } 4.0 \\
\mu \mathrm{mol} \mathrm{cm} \mathrm{cm}^{-2} \mathrm{~h}^{-1}\end{array}$ & 1.23 & $0.1 \mathrm{M} \mathrm{KOH}$ & $\begin{array}{c}100 \mathrm{~mW} \mathrm{~cm}^{-2}, \mathrm{AM} \\
1.5 \mathrm{G} \text { (Newport) }\end{array}$ & 37 \\
\hline $\begin{array}{c}\mathrm{CN}_{\mathrm{TM}} \\
\text { photoanode }\end{array}$ & $\begin{array}{l}\text { Seeded crystallization } \\
\text { of CN monomers, } \\
\text { CVD-like process }\end{array}$ & 353 & $\begin{array}{c}\mathrm{H}_{2}: \text { ca. } 1.88 \\
\mu \mathrm{mol} \mathrm{cm}^{-2} \mathrm{~h}^{-1} \\
\mathrm{O}_{2}: \text { ca. } 0.91 \\
\mu \mathrm{mol} \mathrm{cm} \mathrm{cm}^{-2} \mathrm{~h}^{-1}\end{array}$ & 1.23 & $0.1 \mathrm{M} \mathrm{KOH}$ & $\begin{array}{c}100 \mathrm{~mW} \mathrm{~cm}^{-2}, \mathrm{AM} \\
1.5 \mathrm{G} \text { (Newport) }\end{array}$ & 3 \\
\hline 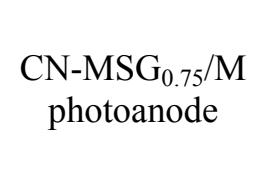 & $\begin{array}{c}\text { Doctor-blade } \\
\text { technique, CVD-like } \\
\text { process }\end{array}$ & 270 & $\begin{array}{c}\mathrm{H}_{2}: \text { ca. } 2.5 \\
\mu \mathrm{mol} \mathrm{cm} \mathrm{cm}^{-2} \mathrm{~h}^{-1} \\
\mathrm{O}_{2}: \text { ca. } 0.3 \\
\mu \mathrm{mol} \mathrm{cm} \mathrm{cm}^{-2} \mathrm{~h}^{-1}\end{array}$ & 1.23 & $0.1 \mathrm{M} \mathrm{KOH}$ & $\begin{array}{c}100 \mathrm{~mW} \mathrm{~cm}{ }^{-2}, \mathrm{AM} \\
1.5 \mathrm{G} \text { (Newport) }\end{array}$ & 38 \\
\hline $\begin{array}{l}\mathrm{P} / \mathrm{B} \text {-layer-doping } \\
\mathrm{C}_{3} \mathrm{~N}_{4} \text { photoanode }\end{array}$ & $\begin{array}{c}\text { Thermal vapour } \\
\text { deposition }\end{array}$ & $150 \pm 10$ & - & 1.23 & $\begin{array}{c}0.1 \mathrm{M} \\
\mathrm{Na}_{2} \mathrm{SO}_{4}\end{array}$ & $\begin{array}{c}300 \mathrm{~W} \text { Xe lamp, } \\
\text { AM } 1.5 \mathrm{G}\end{array}$ & 39 \\
\hline $\mathrm{CN}-\mathrm{MeM} / \mathrm{M}_{0.20}$ & $\begin{array}{c}\text { Doctor-blade } \\
\text { technique, CVD-like } \\
\text { process }\end{array}$ & $\begin{array}{c}133 \\
383 \text { with } \\
\text { TEOA }\end{array}$ & $\begin{array}{l}\mathrm{H}_{2} \text { : ca. } 0.28 \\
\text { mmol g-1 } \mathrm{h}^{-1} \\
\text { with TEOA }\end{array}$ & 1.23 & $0.1 \mathrm{M} \mathrm{KOH}$ & $\begin{array}{c}100 \mathrm{~mW} \mathrm{~cm}^{-2}, \mathrm{AM} \\
1.5 \mathrm{G} \text { (Newport) }\end{array}$ & 40 \\
\hline $\begin{array}{l}\text { In situ grown } \\
\text { porous CN/rGO } \\
\text { films }\end{array}$ & $\begin{array}{c}\text { Doctor-blade } \\
\text { technique, CVD-like } \\
\text { process }\end{array}$ & $\begin{array}{c}124.5 \\
272 \text { with } \\
\text { TEOA }\end{array}$ & $\begin{array}{c}\mathrm{H}_{2}: 0.76 \\
\text { mmol g-1 } \mathrm{h}^{-1} \\
\text { with TEOA }\end{array}$ & 1.23 & $0.1 \mathrm{M} \mathrm{KOH}$ & $\begin{array}{c}100 \mathrm{~mW} \mathrm{~cm}{ }^{-2}, \mathrm{AM} \\
1.5 \mathrm{G} \text { (Newport) }\end{array}$ & 41 \\
\hline PCN photoanode & $\begin{array}{l}\text { Molten mediate } \\
\text { polymerization }\end{array}$ & ca. 110 & - & 1.23 & $\begin{array}{l}1.0 \mathrm{M} \\
\mathrm{NaOH}\end{array}$ & $\begin{array}{c}100 \mathrm{~mW} \mathrm{~cm}^{-2}, \mathrm{AM} \\
1.5 \mathrm{G} \mathrm{(Newport)}\end{array}$ & 42 \\
\hline $\begin{array}{c}\mathrm{CN}-\mathrm{U}_{10} \mathrm{M}_{0.5} \\
\text { photoelectrode }\end{array}$ & $\begin{array}{l}\text { Sprayed a seeding } \\
\text { layer, CVD-like } \\
\text { process }\end{array}$ & ca. 110 & - & 1.23 & $0.1 \mathrm{M} \mathrm{KOH}$ & $\begin{array}{c}100 \mathrm{~mW} \mathrm{~cm}{ }^{-2}, \mathrm{AM} \\
1.5 \mathrm{G} \text { (Newport) }\end{array}$ & 43 \\
\hline
\end{tabular}


Table S3. Photoelectrochemical performances of the heterostructure PCN photoanodes.

\begin{tabular}{|c|c|c|c|c|c|c|c|}
\hline Catalyst & Preparation method & $\begin{array}{c}\text { Photocurrent } \\
\text { density }(\mu \mathrm{A} \\
\left.\mathrm{cm}^{-2}\right)\end{array}$ & $\begin{array}{l}\text { Gas } \\
\text { production } \\
\text { rate }\end{array}$ & $\begin{array}{c}\text { Potential } \\
\text { vs RHE (V) }\end{array}$ & Electrolyte & Light source & $\begin{array}{c}\text { Corresponding } \\
\text { Author (Ref.) }\end{array}$ \\
\hline $\begin{array}{c}\text { Boron-doped CN } \\
\text { films }\end{array}$ & $\begin{array}{l}\text { Rapid thermal vapour } \\
\text { deposition }\end{array}$ & 103.2 & - & 1.23 & $\begin{array}{c}0.1 \mathrm{M} \\
\mathrm{Na}_{2} \mathrm{SO}_{4}\end{array}$ & $\begin{array}{c}150 \mathrm{~W} \text { Xe lamp, } \\
\text { AM } 1.5 \mathrm{G}\end{array}$ & 44 \\
\hline $\begin{array}{l}\mathrm{CN}^{\mathrm{C}}-\mathrm{CM}_{120} \mathrm{M} \\
\text { electrode }\end{array}$ & $\begin{array}{c}\text { Electrophoretic } \\
\text { deposition, CVD-like } \\
\text { process }\end{array}$ & $\begin{array}{l}\text { ca. } 47 \\
122 \text { with } \\
\text { TEOA }\end{array}$ & $\begin{array}{l}\mathrm{H}_{2}: 0.55 \\
\mathrm{mmol} \mathrm{g}^{-1} \mathrm{~h}^{-1} \\
\text { with TEOA }\end{array}$ & 1.23 & $0.1 \mathrm{M} \mathrm{KOH}$ & $\begin{array}{c}100 \mathrm{~mW} \mathrm{~cm} \mathrm{~cm}^{-2}, \mathrm{AM} \\
1.5 \mathrm{G} \text { (Newport) }\end{array}$ & 45 \\
\hline
\end{tabular}


Table S4. Photoelectrochemical performances of the heterostructure photoelectrode formed by integrating metal-free with metal oxides.

\begin{tabular}{|c|c|c|c|c|c|c|c|c|}
\hline Catalyst & $\begin{array}{l}\text { Metal-free } \\
\text { materials }\end{array}$ & Preparation method & $\begin{array}{c}\text { Photocurrent } \\
\text { density (mA } \\
\left.\mathrm{cm}^{-2}\right) \\
\end{array}$ & $\begin{array}{c}\text { Gas } \\
\text { production } \\
\text { rate }\end{array}$ & $\begin{array}{c}\text { Potential } \\
\text { vs RHE (V) }\end{array}$ & Electrolyte & Light source & $\begin{array}{l}\text { Corresponding } \\
\text { Author (Ref.) }\end{array}$ \\
\hline PCN@ZnO & $\mathrm{PCN}$ & Dip-coating process & $12.2 \mu \mathrm{A}$ & - & 1.5 Vvs. SCE & $\begin{array}{c}0.1 \mathrm{M} \\
\mathrm{Na}_{2} \mathrm{SO}_{4}\end{array}$ & $\begin{array}{c}500 \mathrm{~W} \text { Xe lamp with } \\
420 \mathrm{~nm} \text { filters }\left(23.6^{\left.\mathrm{mW} \mathrm{cm}^{-2}\right)}\right.\end{array}$ & 46 \\
\hline $\begin{array}{c}\mathrm{ZnO} / \mathrm{g}-\mathrm{C}_{\mathrm{x}} \mathrm{N}_{\mathrm{y}} \\
\text { (1.0) photoanode }\end{array}$ & $\mathrm{g}-\mathrm{C}_{\mathrm{x}} \mathrm{N}_{\mathrm{y}}$ & $\begin{array}{l}\text { Hydrothermal method, dip- } \\
\text { coating, thermal } \\
\text { polymerization }\end{array}$ & 0.25 & - & 1.23 & $0.1 \mathrm{M} \mathrm{KOH}$ & $\begin{array}{c}\text { Solar simulator } \\
\text { (WACOM, class } \\
\text { AAA) }\end{array}$ & 47 \\
\hline Y:ZnO@PCN & $\mathrm{PCN}$ & $\begin{array}{l}\text { Hydrothermal method, } \\
\text { thermal vapor condensation }\end{array}$ & 0.4 & - & 1.23 & $\begin{array}{c}0.5 \mathrm{M} \\
\mathrm{Na}_{2} \mathrm{SO}_{4}\end{array}$ & $\begin{array}{c}100 \mathrm{~mW} \mathrm{~cm} \mathrm{~cm}^{-2}, \mathrm{AM} \\
1.5 \mathrm{G} \text { (Newport) }\end{array}$ & 48 \\
\hline $\begin{array}{l}\mathrm{Co}_{3} \mathrm{O}_{4} / \mathrm{P}- \\
\mathrm{PCN} / \mathrm{TiO}_{2}\end{array}$ & P- doped PCN & $\begin{array}{l}\text { Hydrothermal method, dip- } \\
\text { coating, thermal } \\
\text { polymerization }\end{array}$ & 1.58 & $\begin{array}{c}\mathrm{H}_{2}: 26.16 \\
\mu \mathrm{mol} \mathrm{cm} \mathrm{cm}^{-2} \mathrm{~h}^{-1}\end{array}$ & 1.23 & $\begin{array}{c}0.5 \mathrm{M} \\
\mathrm{Na}_{2} \mathrm{SO}_{4}\end{array}$ & $\begin{array}{l}150 \text { W Xe lamp, } \\
\text { AM 1.5G }\end{array}$ & 49 \\
\hline $\begin{array}{c}\mathrm{P}-\mathrm{PCN} / \mathrm{TiO}_{2} \\
\mathrm{NTs}\end{array}$ & P- doped PCN & Wet-dipping method & 1.98 & $\begin{array}{c}\mathrm{H}_{2}: 36.6 \mu \mathrm{mol} \\
\mathrm{cm}^{-2} \mathrm{~h}^{-1}\end{array}$ & 1.0 & $\begin{array}{r}1.0 \mathrm{M} \\
\mathrm{NaOH}\end{array}$ & $\begin{array}{c}300 \mathrm{~W} \text { Xe lamp, } \\
\text { simulated solar light } \\
\text { source }\left(\begin{array}{c}100 \mathrm{~mW} \mathrm{~cm}^{-} \\
2\end{array}\right)\end{array}$ & 50 \\
\hline $\mathrm{CDs} / \mathrm{UCN} / \mathrm{TiO}_{2}$ & $\mathrm{UCN}$ & $\begin{array}{l}\text { Hydrothermal method, } \\
\text { thermal polycondensation } \\
\text { method }\end{array}$ & 1.43 & $\begin{array}{c}\mathrm{H}_{2}: 49.8 \mu \mathrm{mol} \\
\mathrm{h}^{-1}\end{array}$ & 1.23 & $\begin{array}{c}1.0 \mathrm{M} \\
\mathrm{NaOH}\end{array}$ & $\begin{array}{c}500 \text { W Xe lamp, } \\
\text { AM 1.5G }\end{array}$ & 51 \\
\hline $\begin{array}{c}\mathrm{TiO}_{2} @ \mathrm{PCNQD} \\
\text { NWs }\end{array}$ & $\begin{array}{l}\text { PCN quantum } \\
\text { dots }\end{array}$ & $\begin{array}{l}\text { Solvothermal method, } \\
\text { thermal polymerization }\end{array}$ & ca. 3.4 & - & 1.0 & $1.0 \mathrm{M} \mathrm{KOH}$ & $\begin{array}{c}\text { Xe lamp, } 300 \mathrm{~mW} \\
\mathrm{~cm}^{-2}\end{array}$ & 52 \\
\hline $\mathrm{PCN} / \mathrm{TiO}_{2}$ & $\mathrm{PCN}$ & $\begin{array}{l}\text { Electrochemical anodization, } \\
\text { CVD method }\end{array}$ & 0.72 & - & 1.23 & $\begin{array}{c}0.1 \mathrm{M} \\
\mathrm{Na}_{2} \mathrm{SO}_{4}\end{array}$ & $\begin{array}{l}300 \mathrm{~W} \text { Xe lamp with } \\
420 \mathrm{~nm} \text { filters }\end{array}$ & 53 \\
\hline
\end{tabular}


Table S4. (continued) Photoelectrochemical performances of the heterostructure photoelectrode formed by integrating metal-free with metal oxides.

\begin{tabular}{|c|c|c|c|c|c|c|c|c|}
\hline Catalyst & $\begin{array}{l}\text { Metal-free } \\
\text { materials }\end{array}$ & Preparation method & $\begin{array}{c}\text { Photocurrent } \\
\text { density (mA } \\
\left.\mathrm{cm}^{-2}\right)\end{array}$ & $\begin{array}{c}\text { Gas } \\
\text { production } \\
\text { rate }\end{array}$ & $\begin{array}{c}\text { Potential } \\
\text { vs RHE (V) }\end{array}$ & Electrolyte & Light source & $\begin{array}{l}\text { Corresponding } \\
\text { Author (Ref.) }\end{array}$ \\
\hline $\begin{array}{l}\mathrm{PCNQD} / \mathrm{TiO}_{2} \\
\text { NTAs }\end{array}$ & $\begin{array}{l}\text { PCN quantum } \\
\text { dots }\end{array}$ & $\begin{array}{l}\text { Anodization process, } \\
\text { immersion method }\end{array}$ & 1.34 & $\begin{array}{c}\mathrm{H}_{2}: 22.0 \mu \mathrm{mol} \\
\mathrm{cm}^{-2} \mathrm{~h}^{-1}\end{array}$ & $\begin{array}{l}0.3 \mathrm{~V} \text { vs } \\
\mathrm{Ag} / \mathrm{AgCl}\end{array}$ & $\begin{array}{c}0.1 \mathrm{M} \\
\mathrm{Na}_{2} \mathrm{SO}_{4}\end{array}$ & $\begin{array}{c}300 \mathrm{~W} \text { Xe lamp, } \\
\text { simulated solar light } \\
\text { source }\left(100 \mathrm{~mW} \mathrm{~cm}^{-}\right. \\
2)\end{array}$ & 54 \\
\hline $\mathrm{TiO}_{2} / \mathrm{P}-\mathrm{PCN}$ & $\mathrm{PCN}$ & $\begin{array}{l}\text { Hydrothermal method, } \\
\text { immersion method }\end{array}$ & 0.3 & - & 1.23 & $\begin{array}{c}1.0 \mathrm{M} \\
\mathrm{NaOH}\end{array}$ & $\begin{array}{c}150 \mathrm{~W} \text { Xe lamp, } \\
\text { AM 1.5G, } \lambda>420 \\
\mathrm{~nm}\end{array}$ & 55 \\
\hline $\mathrm{TCN} / \mathrm{TiO}_{2} \mathrm{NRs}$ & $\mathrm{TCN}$ & $\begin{array}{l}\text { Hydrothermal method, } \\
\text { thermal polymerization }\end{array}$ & 15 & $\begin{array}{l}\mathrm{O}_{2}: 0.09 \\
\mathrm{mmol} \mathrm{h}^{-1}\end{array}$ & 1.2 & $\begin{array}{c}0.5 \mathrm{M} \\
\mathrm{KHCO}_{3}\end{array}$ & $\begin{array}{l}50 \mathrm{~W} \text { LED of } 395 \\
\mathrm{~nm}\end{array}$ & 56 \\
\hline $\begin{array}{c}\mathrm{Ti}: \mathrm{Fe}_{2} \mathrm{O}_{3} @ \mathrm{PCN}- \\
\mathrm{CQD}\end{array}$ & $\mathrm{PCN}$ & $\begin{array}{l}\text { Hydrothermal method, } \\
\text { chemical vapor phase } \\
\text { deposition method }\end{array}$ & 3.38 & $\begin{array}{c}\mathrm{H}_{2}: 27.0 \mu \mathrm{mol} \\
\mathrm{h}^{-1} \\
\mathrm{O}_{2}: 11.9 \mu \mathrm{mol} \\
\mathrm{h}^{-1}\end{array}$ & 1.23 & $1.0 \mathrm{M} \mathrm{KOH}$ & $\begin{array}{c}300 \mathrm{~W} \text { Xe lamp, } \\
\text { AM 1.5G (100 mW } \\
\left.\mathrm{cm}^{-2}\right)\end{array}$ & 57 \\
\hline$\alpha-\mathrm{Fe}_{2} \mathrm{O}_{3} / \mathrm{PCN}$ & $\mathrm{PCN}$ & $\begin{array}{l}\text { Aerosol-assisted chemical } \\
\text { vapour deposition, spin } \\
\text { coating technique }\end{array}$ & 1.17 & - & $\begin{array}{l}1.2 \mathrm{~V} \mathrm{vs} \\
\mathrm{Ag} / \mathrm{AgCl}\end{array}$ & $\begin{array}{c}0.5 \mathrm{M} \\
\mathrm{Na}_{2} \mathrm{SO}_{4}\end{array}$ & $\begin{array}{c}\text { Xe lamp, simulated } \\
\text { sunlight }(100 \mathrm{~mW} \\
\left.\mathrm{cm}^{-2}\right)\end{array}$ & 58 \\
\hline $\begin{array}{c}\text { CoNi-tPCN/a- } \\
\mathrm{Fe}_{2} \mathrm{O}_{3}\end{array}$ & $\mathrm{tPCN}$ & $\begin{array}{l}\text { Radio frequency }(\mathrm{RF})- \\
\text { magnetron sputtering, drop- } \\
\text { casting technique }\end{array}$ & 2.73 & - & 1.23 & $\begin{array}{r}1.0 \mathrm{M} \\
\mathrm{NaOH}\end{array}$ & $\begin{array}{c}150 \mathrm{~W} \text { Xe lamp with } \\
420 \mathrm{~nm} \text { filters }(100 \\
\left.\mathrm{mW} \mathrm{cm}^{-2}\right)\end{array}$ & 59 \\
\hline $\begin{array}{c}\mathrm{NiFeO}_{\mathrm{x}} / \mathrm{B}- \\
\mathrm{PCNMo}-\mathrm{BiVO}_{4}\end{array}$ & B doped PCN & $\begin{array}{l}\text { Electrochemical deposition, } \\
\text { dip-coating method }\end{array}$ & 5.93 & $\begin{array}{c}\mathrm{H}_{2}: 77.5 \mu \mathrm{mol} \\
\mathrm{h}^{-1} \\
\mathrm{O}_{2}: \begin{array}{c}33.6 \mu \mathrm{mol} \\
\mathrm{h}^{-1}\end{array} \\
\end{array}$ & 1.23 & $\begin{array}{l}\text { PPB } \\
\text { solution } \\
(\mathrm{pH} 7)\end{array}$ & $\begin{array}{c}100 \mathrm{~mW} \mathrm{~cm}^{-2}, \mathrm{AM} \\
1.5 \mathrm{G} \mathrm{(Newport)}\end{array}$ & 6 \\
\hline
\end{tabular}


Table S4. (continued) Photoelectrochemical performances of the heterostructure photoelectrode formed by integrating metal-free with metal oxides.

\begin{tabular}{|c|c|c|c|c|c|c|c|c|}
\hline Catalyst & $\begin{array}{l}\text { Metal-free } \\
\text { materials }\end{array}$ & Preparation method & $\begin{array}{c}\text { Photocurrent } \\
\text { density (mA } \\
\left.\mathrm{cm}^{-2}\right)\end{array}$ & $\begin{array}{c}\text { Gas } \\
\text { production } \\
\text { rate }\end{array}$ & $\begin{array}{c}\text { Potential } \\
\text { vs RHE (V) }\end{array}$ & Electrolyte & Light source & $\begin{array}{l}\text { Corresponding } \\
\text { Author (Ref.) }\end{array}$ \\
\hline $\begin{array}{c}\mathrm{PCN} / \mathrm{BiVO}_{4}^{-} \\
\mathrm{FeOOH} / \mathrm{NiOOH}\end{array}$ & $\mathrm{PCN}$ & $\begin{array}{l}\text { Electrochemical deposition, } \\
\text { dip-coating method }\end{array}$ & 4.87 & - & 1.23 & $\begin{array}{c}0.1 \mathrm{M} \mathrm{KPi} \\
\text { buffer } \\
\text { solution } \\
(\mathrm{pH} \mathrm{7)}\end{array}$ & $\begin{array}{c}300 \text { W Xe lamp, } \\
\text { AM 1.5G (100 mW } \\
\left.\mathrm{cm}^{-2}\right)\end{array}$ & 60 \\
\hline $\begin{array}{c}\mathrm{Co}- \\
\mathrm{Pi} / \mathrm{PCN}^{-} \mathrm{BiVO}_{4}\end{array}$ & $\mathrm{PCN}$ & $\begin{array}{l}\text { Electrochemical deposition, } \\
\text { electrophoresis process }\end{array}$ & 3.24 & $\begin{array}{c}\mathrm{H}_{2}: 51.8 \mu \mathrm{mol} \\
\mathrm{cm}^{-2} \mathrm{~h}^{-1} \\
\mathrm{O}_{2}: 25.5 \mu \mathrm{mol} \\
\mathrm{cm}^{-2} \mathrm{~h}^{-1}\end{array}$ & 1.23 & $\begin{array}{l}1.0 \mathrm{M} \mathrm{PB} \\
\text { buffer } \\
\text { solution } \\
(\mathrm{pH} 7)\end{array}$ & $\begin{array}{c}300 \text { W Xe lamp, } \\
\text { AM 1.5G (100 mW } \\
\left.\mathrm{cm}^{-2}\right)\end{array}$ & 61 \\
\hline
\end{tabular}


Table S5. Photoelectrochemical performances by using metal-free materials as conductors.

\begin{tabular}{|c|c|c|c|c|c|c|c|}
\hline Catalyst & $\begin{array}{c}\text { Metal-free } \\
\text { materials as } \\
\text { conductive layers }\end{array}$ & $\begin{array}{l}\text { Preparation } \\
\text { method }\end{array}$ & $\begin{array}{c}\text { Photocurrent } \\
\text { density }(\mu \mathrm{A} \\
\left.\mathrm{cm}^{-2}\right)\end{array}$ & $\begin{array}{l}\text { Potential } \\
\text { vs RHE } \\
\text { (V) }\end{array}$ & Electrolyte & Light source & $\begin{array}{c}\text { Corresponding } \\
\text { Author (Ref.) }\end{array}$ \\
\hline $\begin{array}{l}\text { CNT film on } \\
\text { carbon paper }\end{array}$ & Carbon paper & $\begin{array}{c}\text { Seeded } \\
\text { crystallization of } \\
\text { CN monomers }\end{array}$ & ca. 15 & 1.23 & $\begin{array}{l}0.1 \mathrm{M} \\
\mathrm{KOH}\end{array}$ & $\begin{array}{c}100 \mathrm{~mW} \mathrm{~cm}^{-2} \\
\text { AM 1.5G } \\
\text { (Newport) }\end{array}$ & 3 \\
\hline $\mathrm{CNC}(\mathrm{m})$ & Carbon paper & $\begin{array}{c}\text { Seeded } \\
\text { crystallization of } \\
\text { CN monomers }\end{array}$ & ca. 19 & 1.23 & $\begin{array}{l}0.1 \mathrm{M} \\
\mathrm{KOH}\end{array}$ & $\begin{array}{l}1 \text { sun } \\
\text { illumination }\end{array}$ & 62 \\
\hline $\begin{array}{c}\text { TF-PCN- } \\
\text { Sol@CNTs }\end{array}$ & Carbon nanotubes & Dispersion method & 17 & $\begin{array}{l}-0.2 \mathrm{~V} \text { vs. } \\
\mathrm{Ag} / \mathrm{AgCl}\end{array}$ & - & $\begin{array}{c}300 \mathrm{~W} \text { Xe lamp } \\
\text { with } 420 \mathrm{~nm} \\
\text { filters }\end{array}$ & 63 \\
\hline CNT-CN & Carbon nanotubes & $\begin{array}{c}\text { Thermal } \\
\text { condensation }\end{array}$ & 8.5 & 1.23 & $\begin{array}{l}0.1 \mathrm{M} \\
\mathrm{KOH}\end{array}$ & $\begin{array}{c}100 \mathrm{~mW} \mathrm{~cm}^{-2} \\
\text { AM } 1.5 \mathrm{G} \\
\text { (Newport) }\end{array}$ & 64 \\
\hline$\alpha-\mathrm{Fe}_{2} \mathrm{O}_{3}-\mathrm{UCDs}$ & Carbon dots & $\begin{array}{l}\text { Hydrothermal } \\
\text { method }\end{array}$ & 0.35 & 1.23 & $\begin{array}{c}1.0 \mathrm{M} \\
\mathrm{NaOH}\end{array}$ & $\begin{array}{c}100 \mathrm{~mW} \mathrm{~cm} \mathrm{~cm}^{-2} \\
\text { AM 1.5G } \\
\text { (Newport) }\end{array}$ & 65 \\
\hline $\mathrm{SnS}_{2} \perp \mathrm{CC}$ & Carbon cloth & $\begin{array}{c}\text { Modified chemical } \\
\text { vapor deposition } \\
\text { method }\end{array}$ & $\begin{array}{c}1.92 \pm 0.01 \\
\mathrm{~mA} \mathrm{~cm}{ }^{-2}\end{array}$ & 1.4 & $\begin{array}{c}0.5 \mathrm{M} \\
\mathrm{Na}_{2} \mathrm{SO}_{4}\end{array}$ & 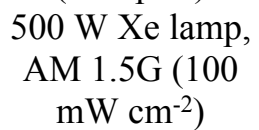 & 7 \\
\hline $\mathrm{MoS}_{2} / \mathrm{Au} @ \mathrm{CFC}$ & Carbon fiber cloth & $\begin{array}{l}\text { Hydrothermal } \\
\text { method }\end{array}$ & $10 \mathrm{~mA} \mathrm{~cm}-2$ & 1.23 & $\begin{array}{c}0.1 \mathrm{M} \\
\mathrm{KH}_{2} \mathrm{PO}_{4}\end{array}$ & 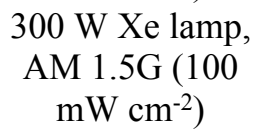 & 66 \\
\hline $\begin{array}{c}\mathrm{CuCo}_{2} \mathrm{~S}_{4} @ \\
\mathrm{CuCo}_{2} \mathrm{O}_{4} / \mathrm{CC}\end{array}$ & Carbon cloth & $\begin{array}{l}\text { Hydrothermal } \\
\text { method }\end{array}$ & ca. 20 & $\begin{array}{l}0.5 \mathrm{~V} \text { vs. } \\
\text { SCE }\end{array}$ & $\begin{array}{l}\text { 1.0 M PB } \\
\text { buffer } \\
\text { solution } \\
(\mathrm{pH} 7)\end{array}$ & $\begin{array}{c}300 \mathrm{~W} \text { Xe lamp } \\
(\lambda>420 \mathrm{~nm})\end{array}$ & 67 \\
\hline
\end{tabular}


Table S6. Photoelectrochemical performances by using metal-free materials as co-catalyst.

\begin{tabular}{|c|c|c|c|c|c|c|c|c|}
\hline Catalyst & $\begin{array}{c}\text { Metal-free } \\
\text { materials as co- } \\
\text { catalyst }\end{array}$ & Preparation method & $\begin{array}{c}\text { Photocurrent } \\
\text { density (mA } \\
\left.\mathrm{cm}^{-2}\right) \\
\end{array}$ & $\begin{array}{c}\text { Gas } \\
\text { production } \\
\text { rate }\end{array}$ & $\begin{array}{c}\text { Potential } \\
\text { vs RHE (V) }\end{array}$ & Electrolyte & Light source & $\begin{array}{l}\text { Corresponding } \\
\text { Author (Ref.) }\end{array}$ \\
\hline $\mathrm{CQDs} / \mathrm{FeOOH} / \mathrm{BiVO}_{4}$ & CQDs & $\begin{array}{l}\text { Spin coating- } \\
\text { pyrolysis method, } \\
\text { spin coating method }\end{array}$ & 2.53 & $\begin{array}{c}\mathrm{H}_{2}: 45.2 \\
\mu \mathrm{mol} \mathrm{h}-1 \\
\mathrm{O}_{2}: 22.1 \\
\mu \mathrm{mol} \mathrm{h}-1\end{array}$ & 1.23 & $\begin{array}{c}0.2 \mathrm{M} \\
\mathrm{Na}_{2} \mathrm{SO}_{4}\end{array}$ & $\begin{array}{c}350 \mathrm{~W} \text { Xe lamp, } \\
\text { AM 1.5G }(100 \mathrm{~mW} \\
\left.\mathrm{cm}^{-2}\right)\end{array}$ & 68 \\
\hline $\mathrm{CQDs} / \mathrm{A} / \mathrm{R}-\mathrm{TiO}_{2}$ & CQDs & $\begin{array}{l}\text { Hydrothermal } \\
\text { method, spin coating } \\
\text { method }\end{array}$ & 2.76 & - & 1.23 & $\begin{array}{c}0.2 \mathrm{M} \\
\mathrm{Na}_{2} \mathrm{SO}_{4}\end{array}$ & $\begin{array}{c}350 \text { W Xe lamp, } \\
\text { AM 1.5G }(100 \mathrm{~mW} \\
\left.\mathrm{cm}^{-2}\right)\end{array}$ & 69 \\
\hline $\mathrm{CQDs} / \mathrm{ZnO}: \mathrm{Ga} / \mathrm{ZnO}$ & CQDs & $\begin{array}{c}\text { Hydrothermal } \\
\text { method, immersion } \\
\text { method }\end{array}$ & 1.5 & - & 1.6 & $\begin{array}{c}0.5 \mathrm{M} \\
\mathrm{Na}_{2} \mathrm{SO}_{4}\end{array}$ & $\begin{array}{c}300 \text { W Xe lamp, } \\
\text { AM 1.5G }(100 \mathrm{~mW} \\
\left.\mathrm{cm}^{-2}\right)\end{array}$ & 70 \\
\hline $\begin{array}{c}\mathrm{Bi}_{2} \mathrm{O}_{4} / \mathrm{Mo}- \\
\mathrm{BiVO} / \mathrm{CQDs} / \mathrm{Ni}- \\
\mathrm{FeOOH}\end{array}$ & CQDs & $\begin{array}{l}\text { Chemical batch } \\
\text { deposition process, } \\
\text { immersion method }\end{array}$ & 6.7 & - & 1.23 & $\begin{array}{l}0.5 \mathrm{M} \mathrm{PB} \\
\text { buffer } \\
\text { solution } \\
\text { (pH 7) }\end{array}$ & $\begin{array}{c}100 \mathrm{~mW} \mathrm{~cm}{ }^{-2}, \mathrm{AM} \\
1.5 \mathrm{G} \text { (Newport) }\end{array}$ & 71 \\
\hline $\mathrm{N}-\mathrm{CDs} @ \alpha-\mathrm{Fe}_{2} \mathrm{O}_{3} / \mathrm{Ti}$ & $\begin{array}{l}\text { N-doped carbon } \\
\text { dots }\end{array}$ & $\begin{array}{l}\text { Hydrothermal } \\
\text { method, immersion } \\
\text { method }\end{array}$ & 0.41 & - & 1.23 & $\begin{array}{l}1.0 \mathrm{M} \\
\mathrm{KOH}\end{array}$ & $\begin{array}{c}300 \mathrm{~W} \text { Xe lamp, } \\
\text { AM 1.5G }(100 \mathrm{~mW} \\
\left.\mathrm{cm}^{-2}\right)\end{array}$ & 73 \\
\hline $\mathrm{BiVO}_{4} / \mathrm{PCN}-\mathrm{NS}$ & PCN nanosheets & $\begin{array}{l}\text { Drop-casting process, } \\
\text { immersion method }\end{array}$ & 3.12 & $\begin{array}{l}\mathrm{H}_{2}: 48.5 \\
\mu \mathrm{mol} \mathrm{h}{ }^{-1}\end{array}$ & 1.23 & $\begin{array}{c}0.1 \mathrm{M} \\
\mathrm{Na}_{2} \mathrm{SO}_{4}\end{array}$ & $\begin{array}{c}300 \mathrm{~W} \text { Xe lamp } \\
\text { with } 420 \mathrm{~nm} \text { filters } \\
\left(200 \mathrm{~mW} \mathrm{~cm}^{-2}\right)\end{array}$ & 74 \\
\hline$\left(\mathrm{BiVO}_{4}: \mathrm{In}\right)-\mathrm{PCNQD}$ & $\begin{array}{l}\text { PCN quantum } \\
\text { dots }\end{array}$ & $\begin{array}{l}\text { Drop-casting process, } \\
\text { immersion method }\end{array}$ & 2.42 & - & 1.23 & $\begin{array}{c}0.5 \mathrm{M} \\
\mathrm{Na}_{2} \mathrm{SO}_{4}\end{array}$ & $\begin{array}{c}100 \mathrm{~mW} \mathrm{~cm}-2, \mathrm{AM} \\
1.5 \mathrm{G}\end{array}$ & 75 \\
\hline $\mathrm{PCN} / \mathrm{BiVO}_{4}$ & PCN hydrogel & $\begin{array}{l}\text { Electrochemical } \\
\text { deposition, drop- } \\
\text { coating method }\end{array}$ & 3.56 & - & 1.23 & $\begin{array}{c}0.01 \mathrm{M} \\
\text { PBS } \\
(\mathrm{pH} \mathrm{7.4)}\end{array}$ & $\begin{array}{c}300 \mathrm{~W} \text { Xe lamp, } \\
\text { AM 1.5G (100 mW } \\
\left.\mathrm{cm}^{-2}\right)\end{array}$ & 76 \\
\hline
\end{tabular}


Table S6. (continued) Summary of PEC performance of the reported metal-free materials as co-catalyst.

\begin{tabular}{|c|c|c|c|c|c|c|c|c|}
\hline Catalyst & $\begin{array}{c}\text { Metal-free } \\
\text { materials as co- } \\
\text { catalyst }\end{array}$ & Preparation method & $\begin{array}{c}\text { Photocurrent } \\
\text { density (mA } \\
\left.\mathrm{cm}^{-2}\right)\end{array}$ & $\begin{array}{c}\text { Gas } \\
\text { production } \\
\text { rate }\end{array}$ & $\begin{array}{c}\text { Potential } \\
\text { vs RHE (V) }\end{array}$ & Electrolyte & Light source & $\begin{array}{l}\text { Corresponding } \\
\text { Author (Ref.) }\end{array}$ \\
\hline $\mathrm{Bi}_{2} \mathrm{MoO}_{6} / \mathrm{PCN}-\mathrm{NS}$ & PCN nanosheets & $\begin{array}{l}\text { Hydrothermal } \\
\text { method, immersion } \\
\text { method }\end{array}$ & 0.52 & - & $\begin{array}{l}0.8 \mathrm{~V} \text { vs. } \\
\text { SCE }\end{array}$ & $\begin{array}{c}0.1 \mathrm{M} \\
\mathrm{Na}_{2} \mathrm{SO}_{4}\end{array}$ & $\begin{array}{c}300 \mathrm{~W} \text { Xe lamp } \\
\text { with } 420 \mathrm{~nm} \text { filters } \\
\left(100 \mathrm{~mW} \mathrm{~cm}^{-2}\right)\end{array}$ & 77 \\
\hline $\mathrm{NiOOH} / \mathrm{BP} / \mathrm{BiVO}_{4}$ & $\begin{array}{c}\text { Black } \\
\text { phosphorene }\end{array}$ & $\begin{array}{c}\text { Electrochemical } \\
\text { deposition, immersion } \\
\text { method }\end{array}$ & 4.48 & $\begin{array}{c}\mathrm{H}_{2}: \text { ca. } 42 \\
\mu \mathrm{mol} \mathrm{h} \\
\mathrm{O}_{2}: \text { ca. }^{-1} \\
\mu \mathrm{mol} \mathrm{h} \\
\end{array}$ & 1.23 & $\begin{array}{c}0.5 \mathrm{M} \mathrm{KPi} \\
\text { buffer } \\
\text { solution } \\
(\mathrm{pH} \mathrm{7.1)}\end{array}$ & $\begin{array}{c}150 \mathrm{~W} \text { Xe lamp, } \\
\text { AM 1.5G (100 mW } \\
\left.\mathrm{cm}^{-2}\right)\end{array}$ & 8 \\
\hline $\mathrm{BP} / \mathrm{TiO}_{2}$ & $\begin{array}{l}\text { Black } \\
\text { phosphorus } \\
\text { nanosheets }\end{array}$ & $\begin{array}{c}\text { Hydrothermal } \\
\text { method, spin coating } \\
\text { method }\end{array}$ & 1.10 & - & 1.23 & $\begin{array}{c}0.2 \mathrm{M} \\
\mathrm{Na}_{2} \mathrm{SO}_{4}\end{array}$ & $\begin{array}{c}300 \mathrm{~W} \text { Xe lamp, } \\
\text { AM 1.5G (100 mW } \\
\left.\mathrm{cm}^{-2}\right)\end{array}$ & 78 \\
\hline $\mathrm{TiO}_{2-\mathrm{x}} / \mathrm{BP}$ & $\begin{array}{l}\text { Black } \\
\text { phosphorus } \\
\text { quantum dot }\end{array}$ & $\begin{array}{l}\text { Anodization process, } \\
\text { spin coating method }\end{array}$ & 1.12 & - & 1.23 & $\begin{array}{c}0.5 \mathrm{M} \\
\mathrm{Na}_{2} \mathrm{SO}_{4}\end{array}$ & $\begin{array}{c}150 \mathrm{~W} \text { Xe lamp, } \\
\text { AM 1.5G }(100 \mathrm{~mW} \\
\left.\mathrm{cm}^{-2}\right)\end{array}$ & 79 \\
\hline $\mathrm{F}-\mathrm{Fe}_{2} \mathrm{O}_{3}-\mathrm{BNQDs}$ & $\begin{array}{l}\text { Boron nitride } \\
\text { quantum dots }\end{array}$ & $\begin{array}{l}\text { Hydrothermal } \\
\text { method, dip-coating } \\
\text { method }\end{array}$ & 2.24 & - & 1.23 & $\begin{array}{l}1.0 \mathrm{M} \\
\mathrm{NaOH}\end{array}$ & $\begin{array}{c}\text { 300W tungsten } \\
\text { halogen lamp } \\
\left(100 \mathrm{~mW} \mathrm{~cm}^{-2}\right)\end{array}$ & 80 \\
\hline $\mathrm{WO}_{3}-\mathrm{BN}$ & $\begin{array}{l}\text { Boron nitride } \\
\text { quantum dots }\end{array}$ & $\begin{array}{l}\text { Hydrothermal } \\
\text { method, chemical } \\
\text { bath deposition }\end{array}$ & 1.63 & - & 1.23 & $\begin{array}{c}0.1 \mathrm{M} \\
\mathrm{Na}_{2} \mathrm{SO}_{4}\end{array}$ & $\begin{array}{c}\text { Tungsten halogen } \\
\text { lamp } \\
\left(100 \mathrm{~mW} \mathrm{~cm}^{-2}\right)\end{array}$ & 81 \\
\hline $\begin{array}{l}\text { phosphorylated PCN } \\
\text { films }\end{array}$ & Phosphate ions & $\begin{array}{l}\text { Molten mediate } \\
\text { polymerization, } \\
\text { immersion method }\end{array}$ & $\begin{array}{l}\text { ca. } 120 \mu \mathrm{A} \\
\mathrm{cm}^{-2}\end{array}$ & - & 1.23 & $\begin{array}{l}1.0 \mathrm{M} \\
\mathrm{NaOH}\end{array}$ & $\begin{array}{c}100 \mathrm{~mW} \mathrm{~cm} \mathrm{~cm}^{-2}, \mathrm{AM} \\
1.5 \mathrm{G} \text { (Newport) }\end{array}$ & 16 \\
\hline
\end{tabular}




\section{REFERENCES}

(1) Kim, J. H.; Lee, J. S. Elaborately Modified BiVO 4 Photoanodes for Solar Water Splitting. Adv. Mater. 2019, 31, 1806938.

(2) Peng, G.; Albero, J.; Garcia, H.; Shalom, M. A Water-Splitting Carbon Nitride Photoelectrochemical Cell with Efficient Charge Separation and Remarkably Low Onset Potential. Angew. Chem. Int. Ed. 2018, 57, 15807-15811.

(3) Qin, J.; Barrio, J.; Peng, G.; Tzadikov, J.; Abisdris, L.; Volokh, M.; Shalom, M. Direct growth of uniform carbon nitride layers with extended optical absorption towards efficient water-splitting photoanodes. Nat. Commun. $2020,11,4701$.

(4) Sick, T.; Hufnagel, A. G.; Kampmann, J.; Kondofersky, I.; Calik, M.; Rotter, J. M.; Evans, A.; Döblinger, M.; Herbert, S.; Peters, K.; Böhm, D.; Knochel, P.; Medina, D. D.; Fattakhova-Rohlfing, D.; Bein, T. Oriented Films of Conjugated 2D Covalent Organic Frameworks as Photocathodes for Water Splitting. J. Am. Chem. Soc. 2018, 140, 2085-2092.

(5) Wei, S.; Zhang, F.; Zhang, W.; Qiang, P.; Yu, K.; Fu, X.; Wu, D.; Bi, S.; Zhang, F. Semiconducting 2D Triazine-Cored Covalent Organic Frameworks with Unsubstituted Olefin Linkages. J. Am. Chem. Soc. 2019, 141, 14272-14279.

(6) Ye, K.-H.; Li, H.; Huang, D.; Xiao, S.; Qiu, W.; Li, M.; Hu, Y.; Mai, W.; Ji, H.; Yang, S. Enhancing photoelectrochemical water splitting by combining work function tuning and heterojunction engineering. Nat. Commun. 2019, 10, 3687.

(7) Liu, G.; Li, Z.; Hasan, T.; Chen, X.; Zheng, W.; Feng, W.; Jia, D.; Zhou, Y.; Hu, P. Vertically aligned two-dimensional $\mathrm{SnS}_{2}$ nanosheets with a strong photon capturing capability for efficient photoelectrochemical water splitting. J. Mater. Chem. A 2017, $5,1989-1995$.

(8) Zhang, K.; Jin, B.; Park, C.; Cho, Y.; Song, X.; Shi, X.; Zhang, S.; Kim, W.; Zeng, H.; Park, J. H. Black phosphorene as a hole extraction layer boosting solar water splitting of oxygen evolution catalysts. Nat. Commun. 2019, $10,2001$.

(9) Li, L.; Yu, Y.; Ye, G. J.; Ge, Q.; Ou, X.; Wu, H.; Feng, D.; Chen, X. H.; Zhang, Y. Black phosphorus field-effect transistors. Nat. Nanotech. 2014, 9, 372-377. 
(10) Zheng, Y.; Yu, Z.; Ou, H.; Asiri, A. M.; Chen, Y.; Wang, X. Black Phosphorus and Polymeric Carbon Nitride Heterostructure for Photoinduced Molecular Oxygen Activation. Adv. Funct. Mater. 2018, 28, 1705407.

(11) Kim, T. W.; Choi, K.-S. Nanoporous BiVO 4 Photoanodes with Dual-Layer Oxygen Evolution Catalysts for Solar Water Splitting. Science 2014, 343, 990.

(12) Jia, Q.; Zhang, S.; Gao, Z.; Yang, P.; Gu, Q. In situ growth of triazine - heptazine based carbon nitride film for efficient (photo)electrochemical performance. Catal. Sci. Technol. 2019, 9, 425-435.

(13) Xiong, W.; Chen, S.; Huang, M.; Wang, Z.; Lu, Z.; Zhang, R. Q. Crystal-Face Tailored Graphitic Carbon Nitride Films for High-Performance Photoelectrochemical Cells. ChemSusChem 2018, 11, 2497-2501.

(14) Guo, B.; Tian, L.; Xie, W.; Batool, A.; Xie, G.; Xiang, Q.; Jan, S. U.; Boddula, R.; Gong, J. R. Vertically Aligned Porous Organic Semiconductor Nanorod Array Photoanodes for Efficient Charge Utilization. Nano Lett. 2018, 18, 5954-5960.

(15) Li, X.; Cheng, Z.; Fang, Y.; Fu, X.; Wang, X. In Situ Synthesis of Phosphorus-Doped Polymeric Carbon Nitride Sheets for Photoelectrochemical Water Oxidation. Solar RRL 2020, 4, 2000168.

(16) Fang, Y.; Li, X.; Wang, X. Phosphorylation of Polymeric Carbon Nitride Photoanodes with Increased Surface Valence Electrons for Solar Water Splitting. ChemSusChem 2019, 12, 2605-2608.

(17) Huang, M.; Wang, H.; Li, W.; Zhao, Y.-L.; Zhang, R.-Q. In situ textured carbon nitride photoanodes with enhanced photoelectrochemical activity by band-gap state modulation. J. Mater. Chem. A2020.

(18) Fang, Y.; Li, X.; Wang, X. Synthesis of Polymeric Carbon Nitride Films with Adhesive Interfaces for Solar Water Splitting Devices. ACS Catal. 2018, 8, 8774-8780.

(19) Xiong, W.; Huang, M.; Huang, F.; Zhang, R.-Q. Colorful carbon nitride based composite films. Appl. Surf. Sci. 2020, $511,145535$.

(20) Lu, X.; Liu, Z.; Li, J.; Zhang, J.; Guo, Z. Novel framework $\mathrm{g}-\mathrm{C}_{3} \mathrm{~N}_{4}$ film as efficient photoanode for photoelectrochemical water splitting. Appl. Catal. B 2017, 209, 657-662. 
(21) Hou, Y.; Wen, Z.; Cui, S.; Feng, X.; Chen, J. Strongly Coupled Ternary Hybrid Aerogels of N-deficient Porous Graphitic- $\mathrm{C}_{3} \mathrm{~N}_{4}$ Nanosheets/N-Doped Graphene/NiFe-Layered Double Hydroxide for Solar-Driven Photoelectrochemical Water Oxidation. Nano Lett. 2016, 16, 2268-2277.

(22) Peng, G.; Volokh, M.; Tzadikov, J.; Sun, J.; Shalom, M. Carbon Nitride/Reduced Graphene Oxide Film with Enhanced Electron Diffusion Length: An Efficient Photo-Electrochemical Cell for Hydrogen Generation. Adv. Energy Mater. $2018,8$.

(23) Zhang, W.; Albero, J.; Xi, L.; Lange, K. M.; Garcia, H.; Wang, X.; Shalom, M. One-Pot Synthesis of Nickel-Modified Carbon Nitride Layers Toward Efficient Photoelectrochemical Cells. ACS Appl. Mater. Inter. 2017, 9, 32667-32677.

(24) Lv, X.; Cao, M.; Shi, W.; Wang, M.; Shen, Y. A new strategy of preparing uniform graphitic carbon nitride films for photoelectrochemical application. Carbon 2017, 117, 343-350.

(25) Zhao, T.; Zhou, Q.; Lv, Y.; Han, D.; Wu, K.; Zhao, L.; Shen, Y.; Liu, S.; Zhang, Y. Ultrafast Condensation of Carbon Nitride on Electrodes with Exceptional Boosted Photocurrent and Electrochemiluminescence. Angew. Chem. Int. Ed. 2020, 59, 11391143.

(26) Liu, J.; Wang, H.; Chen, Z. P.; Moehwald, H.; Fiechter, S.; van de Krol, R.; Wen, L.; Jiang, L.; Antonietti, M. Microcontact-Printing-Assisted Access of Graphitic Carbon Nitride Films with Favorable Textures toward Photoelectrochemical Application. Adv. Mater. 2015, 27, 712-718.

(27) Peng, G.; Xing, L.; Barrio, J.; Volokh, M.; Shalom, M. A General Synthesis of Porous Carbon Nitride Films with Tunable Surface Area and Photophysical Properties. Angew. Chem. Int. Ed. 2018, 57, 1186-1192.

(28) Wang, R.; Liu, H.; Fan, Z.; Li, L.; Cai, Y.; Xu, G.; Luo, W.; Yang, B.; Zhou, Y.; Zou, Z. Unconventional gas-based bottom-up, meter-area-scale fabrication of hydrogen-bond free g-CN nanorod arrays and coupling layers with $\mathrm{TiO}_{2}$ toward highefficiency photoelectrochemical performance. Nanoscale 2018, 10,3342-3349.

(29) Gu, Q.; Gong, X.; Jia, Q.; Liu, J.; Gao, Z.; Wang, X.; Long, J.; Xue, C. Compact carbon nitride based copolymer films with controllable thickness for photoelectrochemical water splitting. J. Mater. Chem. A 2017, 5, 19062-19071. 
(30) Li, J.; Lei, N.; Hao, H.; Zhou, J. A series of BCN nanosheets with enhanced photoelectrochemical performances. Chem. Phys. Lett. 2017, 672, 99-104.

(31) Fang, Y.; Merenkov, I. S.; Li, X.; Xu, J.; Lin, S.; Kosinova, M. L.; Wang, X. Vertically aligned 2D carbon doped boron nitride nanofilms for photoelectrochemical water oxidation. J. Mater. Chem. A 2020, 8, 13059-13064.

(32) Giusto, P.; Arazoe, H.; Cruz, D.; Lova, P.; Heil, T.; Aida, T.; Antonietti, M. Boron Carbon Nitride Thin Films: From Disordered to Ordered Conjugated Ternary Materials. J. Am. Chem. Soc. 2020, 142, 20883-20891.

(33) Bornoz, P.; Prévot, M. S.; Yu, X.; Guijarro, N.; Sivula, K. Direct Light-Driven Water Oxidation by a Ladder-Type Conjugated Polymer Photoanode. J. Am. Chem. Soc. 2015, 137, 15338-15341.

(34) Dai, C.; He, T.; Zhong, L.; Liu, X.; Zhen, W.; Xue, C.; Li, S.; Jiang, D.; Liu, B. 2,4,6-Triphenyl-1,3,5-Triazine Based Covalent Organic Frameworks for Photoelectrochemical $\mathrm{H}_{2}$ Evolution. Adv. Mater. Interfaces 2021, 8, 2002191.

(35) Kochergin, Y. S.; Beladi-Mousavi, S. M.; Khezri, B.; Lyu, P.; Bojdys, M. J.; Pumera, M. Organic photoelectrode engineering: accelerating photocurrent generation via donor - acceptor interactions and surface-assisted synthetic approach. $J$. Mater. Chem. A 2021, 9, 7162-7171.

(36) Zhang, T.; Hou, Y.; Dzhagan, V.; Liao, Z.; Chai, G.; Löffler, M.; Olianas, D.; Milani, A.; Xu, S.; Tommasini, M.; Zahn, D. R. T.; Zheng, Z.; Zschech, E.; Jordan, R.; Feng, X. Copper-surface-mediated synthesis of acetylenic carbon-rich nanofibers for active metal-free photocathodes. Nat. Commun. 2018, 9, 1140.

(37) Karjule, N.; Singh, C.; Barrio, J.; Tzadikov, J.; Liberman, I.; Volokh, M.; Palomares, E.; Hod, I.; Shalom, M. Carbon Nitride-Based Photoanode with Enhanced Photostability and Water Oxidation Kinetics. Adv. Funct. Mater. $2021,2101724$.

(38) Karjule, N.; Barrio, J.; Xing, L.; Volokh, M.; Shalom, M. Highly Efficient Polymeric Carbon Nitride Photoanode with Excellent Electron Diffusion Length and Hole Extraction Properties. Nano Lett. 2020, 20, 4618-4624.

(39) Luan, P.; Meng, Q.; Wu, J.; Li, Q.; Zhang, X.; Zhang, Y.; O'Dell, L. A.; Raga, S. R.; Pringle, J.; Griffith, J. C.; Sun, C.; Bach, U.; Zhang, J. Unique Layer-Doping-Induced Regulation of Charge Behavior in Metal-Free Carbon Nitride Photoanodes for Enhanced Performance. ChemSusChem 2020, 13, 328-333. 
(40) Xia, J.; Karjule, N.; Abisdris, L.; Volokh, M.; Shalom, M. Controllable Synthesis of Carbon Nitride Films with Type-II Heterojunction for Efficient Photoelectrochemical Cells. Chem. Mater. 2020, 32, 5845-5853.

(41) Peng, G.; Qin, J.; Volokh, M.; Liu, C.; Shalom, M. Graphene oxide in carbon nitride: from easily processed precursors to a composite material with enhanced photoelectrochemical activity and long-term stability. J. Mater. Chem. A 2019, 7, 1171811723.

(42) Fang, Y.; Li, X.; Wang, Y.; Giordano, C.; Wang, X. Gradient sulfur doping along polymeric carbon nitride films as visible light photoanodes for the enhanced water oxidation. Appl. Catal. B 2020, 268, 118398.

(43) Tashakory, A.; Karjule, N.; Abisdris, L.; Volokh, M.; Shalom, M. Mediated Growth of Carbon Nitride Films via SprayCoated Seeding Layers for Photoelectrochemical Applications. Adv. Sustain. Syst. 2021, 2100005.

(44) Ruan, Q.; Luo, W.; Xie, J.; Wang, Y.; Liu, X.; Bai, Z.; Carmalt, C. J.; Tang, J. A Nanojunction Polymer Photoelectrode for Efficient Charge Transport and Separation. Angew. Chem. Int. Ed. 2017, 56, 8221-8225.

(45) Abisdris, L.; Tzadikov, J.; Karjule, N.; Azoulay, A.; Volokh, M.; Shalom, M. Electrophoretic deposition of supramolecular complexes for the formation of carbon nitride films. Sustain. Energ. Fuels 2020, 4, 3879-3883.

(46) Wang, J.; Yang, Z.; Gao, X.; Yao, W.; Wei, W.; Chen, X.; Zong, R.; Zhu, Y. Core-shell g-C3N4@ZnO composites as photoanodes with double synergistic effects for enhanced visible-light photoelectrocatalytic activities. Appl. Catal. B 2017, 217, 169180 .

(47) Hajduk, Š.; Berglund, S. P.; Podlogar, M.; Dražić, G.; Abdi, F. F.; Orel, Z. C.; Shalom, M. Conformal Carbon Nitride Coating as an Efficient Hole Extraction Layer for ZnO Nanowires-Based Photoelectrochemical Cells. Adv. Mater. Interfaces 2017, 4, 1700924.

(48) Fang, Y.; Xu, Y.; Li, X.; Ma, Y.; Wang, X. Coating Polymeric Carbon Nitride Photoanodes on Conductive Y:ZnO Nanorod Arrays for Overall Water Splitting. Angew. Chem. Int. Ed. 2018, 57, 9749-9753. 
(49) Yu, Z.; Li, Y.; Qu, J.; Zheng, R.; Cairney, J. M.; Zhang, J.; Zhu, M.; Khan, A.; Li, W. Enhanced photoelectrochemical water-splitting performance with a hierarchical heterostructure: $\mathrm{Co}_{3} \mathrm{O}_{4}$ nanodots anchored $\mathrm{TiO}_{2} @ \mathrm{P}-\mathrm{C}_{3} \mathrm{~N}_{4}$ core-shell nanorod arrays. Chem. Eng. J. 2021, 404, 126458.

(50) Su, J.; Geng, P.; Li, X.; Zhao, Q.; Quan, X.; Chen, G. Novel phosphorus doped carbon nitride modified TiO2 nanotube arrays with improved photoelectrochemical performance. Nanoscale 2015, 7, 16282-16289.

(51) Kong, W.; Zhang, X.; Chang, B.; Guo, Y.; Li, Y.; Zhang, S.; Yang, B. $\mathrm{TiO}_{2}$ Nanorods Co-decorated with Metal-Free Carbon Materials for Boosted Photoelectrochemical Water Oxidation. ChemElectroChem 2020, 7, $792-799$.

(52) An, T.; Tang, J.; Zhang, Y.; Quan, Y.; Gong, X.; Al-Enizi, A. M.; Elzatahry, A. A.; Zhang, L.; Zheng, G. Photoelectrochemical Conversion from Graphitic $\mathrm{C}_{3} \mathrm{~N}_{4}$ Quantum Dot Decorated Semiconductor Nanowires. ACS Appl. Mater. Inter. 2016, 8, 12772-12779.

(53) Xiao, L.; Liu, T.; Zhang, M.; Li, Q.; Yang, J. Interfacial Construction of Zero-Dimensional/One-Dimensional g-C $\mathrm{N}_{4}$ Nanoparticles $/ \mathrm{TiO}_{2}$ Nanotube Arrays with Z-Scheme Heterostructure for Improved Photoelectrochemical Water Splitting. ACS Sustain. Chem. Eng. 2019, 7, 2483-2491.

(54) Su, J.; Zhu, L.; Chen, G. Ultrasmall graphitic carbon nitride quantum dots decorated self-organized $\mathrm{TiO}_{2}$ nanotube arrays with highly efficient photoelectrochemical activity. Appl. Catal. B 2016, 186, 127-135.

(55) Qin, D.-D.; Quan, J.-J.; Duan, S.-F.; San Martin, J.; Lin, Y.; Zhu, X.; Yao, X.-Q.; Su, J.-Z.; Rodríguez-Gutiérrez, I.; Tao, C.-L.; Yan, Y. High-Performance Photoelectrochemical Water Oxidation with Phosphorus-Doped and Metal Phosphide Cocatalyst-Modified g- $\mathrm{C}_{3} \mathrm{~N}_{4}$ Formation Through Gas Treatment. ChemSusChem 2019, 12, 898-907.

(56) Ma, Y.; Fang, Y.; Fu, X.; Wang, X. Photoelectrochemical conversion of $\mathrm{CO}_{2}$ into $\mathrm{HCOOH}$ using a polymeric carbon nitride photoanode and Cu cathode. Sustain. Energ. Fuels 2020, 4, 5812-5817.

(57) Yi, S.-S.; Yan, J.-M.; Jiang, Q. Carbon quantum dot sensitized integrated $\mathrm{Fe}_{2} \mathrm{O}_{3} @ g-\mathrm{C}_{3} \mathrm{~N}_{4}$ core - shell nanoarray photoanode towards highly efficient water oxidation. J. Mater. Chem. A 2018, 6, 9839-9845. 
(58) Arzaee, N. A.; Mohamad Noh, M. F.; Mohd Ita, N. S. H.; Mohamed, N. A.; Mohd Nasir, S. N. F.; Nawas Mumthas, I. N.; Ismail, A. F.; Mat Teridi, M. A. Nanostructure-assisted charge transfer in $\alpha-\mathrm{Fe}_{2} \mathrm{O}_{3} / g-\mathrm{C}_{3} \mathrm{~N}_{4}$ heterojunctions for efficient and highly stable photoelectrochemical water splitting. Dalton T. 2020, 49, 11317-11328.

(59) Arora, P.; Singh, A. P.; Mehta, B. R.; Basu, S. Metal doped tubular carbon nitride $\left(\mathrm{tC}_{3} \mathrm{~N}_{4}\right)$ based hematite photoanode for enhanced photoelectrochemical performance. Vacuum 2017, 146, 570-577.

(60) Luan, P.; Zhang, Y.; Zhang, X.; Li, Z.; Prathapan, R.; Bach, U.; Zhang, J. Bismuth Vanadate with Electrostatically Anchored 3D Carbon Nitride Nano-networks as Efficient Photoanodes for Water Oxidation. ChemSusChem 2018, 11, $2510-2516$.

(61) Su, J.; Bai, Z.; Huang, B.; Quan, X.; Chen, G. Unique three dimensional architecture using a metal-free semiconductor cross-linked bismuth vanadate for efficient photoelectrochemical water oxidation. Nano Energy 2016, 24, 148-157.

(62) Peng, G.; Qin, J.; Volokh, M.; Shalom, M. Freestanding Hierarchical Carbon Nitride/Carbon-Paper Electrode as a Photoelectrocatalyst for Water Splitting and Dye Degradation. ACS Appl. Mater. Inter. 2019, 11, 29139-29146.

(63) Zhang, J.; Zhang, M.; Lin, L.; Wang, X. Sol Processing of Conjugated Carbon Nitride Powders for Thin-Film Fabrication. Angew. Chem. Int. Ed. 2015, 54, 6297-6301.

(64) Karjule, N.; Rana, M.; Shalom, M.; Barrio, J.; Vilatela, J. J. Controlled Nucleation and Growth of Carbon Nitride Films on CNT Fiber Fabric for Photoelectrochemical Applications. Adv. Sustain. Syst. 2021, 2000265.

(65) Guo, Q.; Luo, H.; Zhang, J.; Ruan, Q.; Prakash Periasamy, A.; Fang, Y.; Xie, Z.; Li, X.; Wang, X.; Tang, J.; Briscoe, J.; Titirici, M.; Jorge, A. B. The role of carbon dots - derived underlayer in hematite photoanodes. Nanoscale 2020, 12, 2022020229.

(66) Xu, X.; Zhou, G.; Dong, X.; Hu, J. Interface Band Engineering Charge Transfer for 3D MoS 2 Photoanode to Boost Photoelectrochemical Water Splitting. ACS Sustain. Chem. Eng. 2017, 5, 3829-3836.

(67) Wang, Q.; Zhang, L. An in situ engineered $\mathrm{CuCo}_{2} \mathrm{~S}_{4} @ \mathrm{CuCo}_{2} \mathrm{O}_{4}$ heterojunction with an O - S interpenetrated interface as a photoanode for selective photoelectrochemical bioanalysis. J. Mater. Chem. A 2020, 8, 9077-9084. 
(68) Zhou, T.; Chen, S.; Wang, J.; Zhang, Y.; Li, J.; Bai, J.; Zhou, B. Dramatically enhanced solar-driven water splitting of $\mathrm{BiVO}_{4}$ photoanode via strengthening hole transfer and light harvesting by co-modification of CQDs and ultrathin $\beta-\mathrm{FeOOH}$ layers.

Chem. Eng. J. 2021, 403, 126350.

(69) Zhou, T.; Chen, S.; Li, L.; Wang, J.; Zhang, Y.; Li, J.; Bai, J.; Xia, L.; Xu, Q.; Rahim, M.; Zhou, B. Carbon quantum dots modified anatase/rutile $\mathrm{TiO}_{2}$ photoanode with dramatically enhanced photoelectrochemical performance. Appl. Catal. B 2020, $269,118776$.

(70) Xiao, J.; Hou, X.; Zhao, L.; Li, Y. A carbon-quantum-dot-sensitized ZnO:Ga/ZnO multijunction composite photoanode for photoelectrochemical water splitting under visible light irradiation. J. Catal. 2017, 346, 70-77.

(71) Ma, M.; Xing, Z.; Zhu, X.; Jiang, P.; Wang, X.; Lin, H.; An, Y.; Su, H.; Yang, S. Interface modulation of BiVO 4 based photoanode with $\mathrm{Bi}(\mathrm{III}) \mathrm{Bi}(\mathrm{V}) \mathrm{O}_{4}$ for enhanced solar water splitting. J. Catal. 2020, 391, 513-521.

(72) Fan, K.; Chen, H.; He, B.; Yu, J. Cobalt polyoxometalate on N-doped carbon layer to boost photoelectrochemical water oxidation of $\mathrm{BiVO}_{4}$. Chem. Eng. J. 2020, 392, 123744.

(73) Huang, X.; Yang, L.; Hao, S.; Zheng, B.; Yan, L.; Qu, F.; Asiri, A. M.; Sun, X. N-Doped carbon dots: a metal-free cocatalyst on hematite nanorod arrays toward efficient photoelectrochemical water oxidation. Inorganic Chemistry Frontiers 2017, 4, 537-540.

(74) Feng, C.; Wang, Z.; Ma, Y.; Zhang, Y.; Wang, L.; Bi, Y. Ultrathin graphitic $\mathrm{C}_{3} \mathrm{~N}_{4}$ nanosheets as highly efficient metalfree cocatalyst for water oxidation. Appl. Catal. B 2017, 205, 19-23.

(75) Shah, A. K.; Sahu, T. K.; Gogoi, D.; Peela, N. R.; Qureshi, M. Surface-engineering of decahedron shaped bismuth vanadate for improved photoelectrochemical water oxidation by indium doping coupled with graphitic carbon nitride quantum dots. $J$. Power Sources 2020, 477, 229024.

(76) Wang, Y.; Jiang, W.; Yao, W.; Liu, Z.; Liu, Z.; Wang, Y.; Shi, L.; Gao, L. BiNV bond: A hole-transfer bridge for highefficient separation and transfer of carriers. J. Colloid Interf. Sci. 2021, 590, 144-153. 
(77) Ma, Y.; Wang, Z.; Jia, Y.; Wang, L.; Yang, M.; Qi, Y.; Bi, Y. Bi $2 \mathrm{MoO}_{6}$ nanosheet array modified with ultrathin graphitic carbon nitride for high photoelectrochemical performance. Carbon 2017, 114, 591-600.

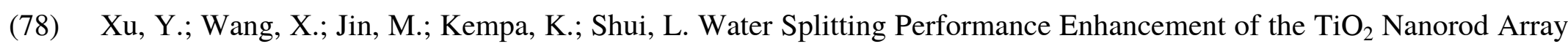
Electrode with Ultrathin Black Phosphorus Nanosheets. ChemElectroChem 2020, 7, 96-104.

(79) Zheng, L.; Ye, X.; Deng, X.; Wang, Y.; Zhao, Y.; Shi, X.; Zheng, H. Black Phosphorus Quantum Dot-Sensitized TiO 2 Nanotube Arrays with Enriched Oxygen Vacancies for Efficient Photoelectrochemical Water Splitting. ACS Sustain. Chem. Eng. 2020, 8, 15906-15914.

(80) Sahu, T. K.; Mohanta, M. K.; Qureshi, M. Modulating water oxidation kinetics utilizing h-BN quantum dots as an efficient hole extractor on fluorine doped hematite photoanode. J. Power Sources 2020, 445, 227341.

(81) Mohanta, M. K.; Sahu, T. K.; Gogoi, D.; Peela, N. R.; Qureshi, M. Hexagonal Boron Nitride Quantum Dots as a Superior Hole Extractor for Efficient Charge Separation in $\mathrm{WO}_{3}$-Based Photoelectrochemical Water Oxidation. ACS Appl. Energ. Mater. 2019, 2, 7457-7466. 